\title{
CIP2A is a prime synthetic-lethal target for BRCA-mutated cancers
}

Salomé Adam ${ }^{1, \dagger}$, Silvia Emma Rossi ${ }^{1, \dagger}$, Nathalie Moatti ${ }^{1}$, Mara De Marco Zompit ${ }^{2}$, Timothy F. Ng ${ }^{1,3}$, Alejandro Álvarez-Quilón', Jessica Desjardins", Vivek Bhaskaran ${ }^{4}$, Giovanni Martino ${ }^{4}$, Dheva Setiaputra $^{1}$, Sylvie M. Noordermeer ${ }^{1 *}$, Toshiro K. Ohsumi ${ }^{4}$, Nicole Hustedt ${ }^{1 \oplus}$, Rachel K. Szilard ${ }^{1}$, Natasha Chaudhary ${ }^{1}$, Meagan Munro ${ }^{1}$, Artur Veloso ${ }^{4}$, Henrique Melo ${ }^{1}$, Shou Yun Yin ${ }^{4}$, Robert Papp ${ }^{4}$, Jordan T.F. Young ${ }^{4}$, Michael Zinda ${ }^{4}$, Manuel Stucki ${ }^{2}$ and Daniel Durocher ${ }^{1,3, *}$

\section{Affiliations:}

1 Lunenfeld-Tanenbaum Research Institute, Mount Sinai Hospital, 600 University Avenue, Toronto, ON, M5G 1X5, Canada

2 Department of Gynecology, University Hospital and University of Zurich, Wagistrasse 14, 8952 Schlieren, Switzerland.

3 Department of Molecular Genetics, University of Toronto, 1 King's College Circle, Toronto, ON, M5S 1A8, Canada

4 Repare Therapeutics, 7210 Frederick-Banting, Suite 100, St-Laurent, QC, H4S 2A1, Canada

$\dagger$ These authors contributed equally to this work

¥Current address: Department of Human Genetics, Leiden University Medical Center, Leiden, The Netherlands.

^ Current address: Ridgeline Therapeutics, Hochbergerstrasse 60C, CH-4057 Basel, Switzerland

* Corresponding author 
BRCA1/2-mutated cancer cells must adapt to the genome instability caused by their deficiency in homologous recombination. Identifying and targeting these adaptive mechanisms may 3 provide new therapeutic strategies. Here we present the results of genome-scale CRISPR/Cas9based synthetic lethality screens in isogenic pairs of BRCA1- and BRCA2-deficient cells that identified the gene encoding CIP2A as essential in a wide range of $B R C A 1$ - and $B R C A 2$-mutated cells. Unlike PARP inhibition, CIP2A-deficiency does not cause accumulation of replicationassociated DNA lesions that require homologous recombination for their repair. CIP2A is cytoplasmic in interphase but, in mitosis, accumulates at DNA lesions as part of a complex with TOPBP1, a multifunctional genome stability factor. In BRCA-deficient cells, the CIP2ATOPBP1 complex prevents lethal mis-segregation of acentric chromosomes that arises from impaired DNA synthesis. Finally, physical disruption of the CIP2A-TOPBP1 complex is highly deleterious in BRCA-deficient cells and tumors, indicating that targeting this mitotic chromosome stability process represents an attractive synthetic-lethal therapeutic strategy for $B R C A 1-$ and $B R C A 2-$ mutated cancers.

The BRCA1 and BRCA2 proteins promote the repair of replication-associated DNA damage by homologous recombination (HR) ${ }^{1}$. Acute inactivation of BRCA2 impedes completion of DNA replication ${ }^{2}$, which is associated with rampant chromosome segregation defects and cell lethality. This phenotype is likely to be shared by BRCA1 since its loss also causes cellular lethality ${ }^{3}$. These observations suggest that during their evolution towards the malignant phenotype, cells with inactivating mutations in $B R C A 1$ and $B R C A 2$ adapt to the replication-associated problems caused by HR deficiency. Identifying the mechanisms that endow cells to complete chromosome duplication and segregation without active HR may thus provide new opportunities for therapeutic intervention. The targeting of these adaptive mechanisms should display efficacy and toxicity profiles that are distinct from genotoxic chemotherapy or poly(ADP-ribose) polymerase (PARP) inhibition, since these latter therapies instead act by increasing the load of DNA lesions that engage HR-dependent DNA repair ${ }^{4}$.

The CIP2A-BRCA synthetic lethality

30 To identify a complement of genes that is essential for the viability of HR-deficient cells, we carried

31 out genome-scale dropout CRISPR-based synthetic lethal screens in isogenic pairs of BRCA1- and

32 BRCA2-mutated cells in the human RPE1-hTERT (immortalized retinal epithelium) and DLD1 (colon 33 adenocarcinoma) backgrounds, respectively (Fig. S1A). We reasoned that genetic interactions 
34 common to the loss of BRCA1 and BRCA2 in two cell lines of different origins would have the

35 potential to uncover universal vulnerabilities to the loss of HR.

37 The screens were carried out with the TKOv2 (BRCA1 screen) or TKOv3 (BRCA2 screen) single38 guide (sg) RNA libraries and were analyzed with a custom-built analysis pipeline, called

39 CRISPRCount Analysis (CCA), dedicated to the identification of synthetic-lethal genetic interactions

40 (see methods), defined here as genes essential for the fitness of a mutated cell line (in this case $B R C A 1^{-}$

$41{ }^{/-}$or $B R C A 2^{-/}$) but not of their isogenic wild type counterparts. CCA identified 55 and 50 genes that 42 selectively impaired fitness in the BRCA1- or BRCA2-mutated cells, respectively (Fig. 1A, Table S1 43 and Datasets S1-S2). The top 10 genes common to both screens were APEX1, APEX2, CHD1L, 44 CHTF8, CIP2A, DSCC1, DDIAS, PARP1, SLC25A28 and XRCC1 (Fig. 1A). Of these, PARP1 and APEX2 are known to display robust synthetic lethal interactions with HR deficiency when depleted or inhibited ${ }^{5-8}$. CHD1L was also recently shown to promote survival to PARP inhibition and to impair fitness of BRCA-deficient cells ${ }^{9,10}$. Other genes with known synthetic lethal interactions with BRCA1/2 such as POLQ ${ }^{11,12}$ or the RNase H2-coding genes ${ }^{6,9}$ were hits in only one of the two cell lines (Table S1).

To identify genetic interactions with highest relevance to the tumor setting, we analyzed the results of two large-scale studies of genetic dependencies in cancer cells lines: the DepMap project ${ }^{13,14}$. We grouped cell lines according to whether or not they harbor biallelic damaging alterations in $B R C A 1$ or $B R C A 2$, and then plotted the distribution of their gene-level depletion scores (where lower numbers indicate negative impact on cell fitness) (Table S2). Despite both datasets having only a few annotated biallelic $B R C A 1$ - or $B R C A 2$-mutated cell lines, $C I P 2 A$ targeting had the most penetrant, significant and profound impact on the fitness of BRCA1/2-deficient cancer cells in both datasets, with APEX2 also showing good separation between the BRCA-proficient and -deficient groups (Fig. 1BC, S1B and Table S2). Since these studies highlighted CIP2A as having a particularly strong genetic interaction with $B R C A 1 / 2$, we then used clonogenic survival assays to confirm the synthetic lethality conferred by the loss of CIP2A in the engineered $B R C A 1^{-/-}$and $B R C A 2^{-/-}$cell lines (Fig. 1D-G, S1CE; details on sgRNA sequences and indel formation are found in Tables S3 \& S4). Re-introduction of an sgRNA-resistant $C I P 2 A$ transgene $\left(C I P 2 A^{*}\right)$ into $B R C A 1^{-/-}$and $B R C A 2^{-/-}$cells rescued the synthetic-lethality phenotype (Fig 1E,G). Lastly, we undertook a "reverse" CRISPR-based synthetic

65 lethality screen with a $C I P 2 A$ knockout query cell line (in the RPE1-hTERT $p 53^{--}$Cas9 background 
PALB2, and FANCM were among the top synthetic-lethal hits, as determined by CCA and BAGEL2

16 (Fig. $1 \mathrm{H}$ and Table $\mathrm{S} 1$ ). We conclude that $C I P 2 A$ is essential in a broad range of engineered and tumor-derived HR-deficient cell lines.

$C I P 2 A$ encodes a protein of 905 amino acid residues that can be broadly split in two regions: a highly structured N-terminal half consisting of an armadillo (Arm) repeat core (residues 1-560) ${ }^{17}$ followed by a C-terminal half predicted to form a coiled-coil ${ }^{17}$ (Fig. 1I). The exact molecular function of CIP2A is unknown although it is a reported inhibitor of the PP2A phosphatase and is overexpressed in multiple tumor types ${ }^{18,19}$. Mice homozygous for a near-null Cip $2 a$ allele produced by gene trapping have a typical lifespan and develop normally with the exception of a mild spermatogenesis defect ${ }^{20}$.

While a direct role for CIP2A in DNA repair or replication has not been reported to date, loss of CIP2A is associated with sensitivity to ATR inhibitors ${ }^{15}$ and to a few other genotoxins ${ }^{21}$, including the TOP1 poison camptothecin (Fig. S2A). These observations initially suggested that CIP2A may repair or prevent accumulation of replication-borne DNA lesions that require HR for their repair, since this is the basis for the PARP1-BRCA and $A P E X 2-B R C A$ synthetic lethality. To test this possibility, we examined spontaneous sister-chromatid exchanges (SCEs), which are reflective of replicationassociated DNA lesions that are repaired by $\mathrm{HR}^{22}$. In contrast to APEX2 $\mathrm{sgRNAs}$ or PARP inhibition 23, CIP2A-depleted cells experience near-basal levels of spontaneous SCEs, indicating that CIP2A loss does not greatly increase the amount of DNA lesions that engage the HR pathway (Figs. 2A and $\mathrm{S} 2 \mathrm{~B})$. In support of this possibility, $C I P 2 A^{-/-}$cells have similar levels of spontaneous DSBs in S phase, marked by $\gamma-\mathrm{H} 2 \mathrm{AX}$, as its parental cell line (Fig. 2B). Together, these results suggest that the CIP2A$B R C A$ synthetic lethality is not due to an increased load of replication-associated DNA lesions that are usually processed by HR.

\section{CIP $2 A$ acts on mitotic DNA lesions}

93 A lack of direct involvement of CIP2A in DNA repair or DNA replication is further supported by the 94 subcellular localization of CIP2A. As previously noted ${ }^{24}$, CIP2A is cytoplasmic in interphase cells as 95 determined by immunofluorescence microscopy (Fig. S2C). DNA damage caused by ionizing 96 radiation (IR) did not promote CIP2A translocation from the cytoplasm to the nucleoplasm in 97 interphase cells, but rather led to a striking formation of IR-induced, $\gamma \mathrm{H} 2 \mathrm{AX}$-colocalising CIP2A foci 98 solely in mitotic cells (Fig. 2C-F). We also observed an increased frequency of mitotic CIP2A foci in 
$B R C A 2^{-/-}$cells over their wild-type counterparts (Figs. 2G and S2D), suggesting that CIP2A responds to DNA damage only during $\mathrm{M}$ phase and that this response is likely relevant to the $C I P 2 A-B R C A$ synthetic lethality.

103 The metaphases of HR-deficient cells treated with PARP inhibitors or depleted of APE2 display 104 increased numbers of radial chromosomes ${ }^{6,25}$, which are likely to be caused by the unscheduled action 105 of non-homologous end-joining on DNA lesions that are normally repaired by HR. Depletion of $106 \mathrm{CIP} 2 \mathrm{~A}$ in $\mathrm{BRCA1}^{-/-}$cells did not increase radial chromosome formation but we did detect an increase 107 in chromatid breaks (Figs. $2 \mathrm{H}$ and S2E). Together with the near-normal SCE frequency of CIP2A $A^{-/-}$ 108 cells, these results further indicate that CIP2A must support the viability of HR-deficient cells via a 109 mechanism distinct from PARP or APE2. A clue to this mechanism emerged when we observed that 110 depletion of CIP2A led to a striking increase in the frequency of micronuclei (MNi) formed in $B R C A 2^{-}$ 111 / cells (Figs. 2I and S2F). These micronuclei were largely CENPA-negative, indicating that they 112 originate from the mis-segregation of acentric (i.e. broken) chromosomes (Fig. 2I). These results 113 suggest that CIP2A promotes the viability of HR-deficient cells by guarding against the formation 114 and/or mis-segregation of acentric chromosomes.

\section{A CIP2A-TOPBP1 complex}

117 The micronucleation caused by loss of CIP2A in HR-deficient cells was revealing in light of work 118 showing that MDC1 and TOPBP1 form mitotic IR-induced foci that promote segregation of broken 119 chromosomes ${ }^{26}$. This was not the sole connection to these genes since analysis of DepMap data ${ }^{13}$ 120 showed essentiality profiles for $M D C 1$ and TOPBP1 that are highly correlated to those of CIP2A (Fig.

$1213 \mathrm{~A}$ ). Similarly, genotoxin sensitivity profiles generated from a DNA damage chemogenomic dataset $122{ }^{21}$ also links CIP2A to MDC1 (Fig. S3A). Together, these data hinted that CIP2A collaborates with

123 MDC1 and TOPBP1 to promote the accurate segregation of damaged chromosomes. In support of 124 this possibility, CIP2A, MDC1 and TOPBP1 colocalized at IR-induced mitotic foci in nocodazole125 treated cells (Fig. 3B). Protein depletion studies with siRNAs further showed that MDC1 was acting 126 upstream of TOPBP1 and CIP2A, and that the localization of TOPBP1 and CIP2A to mitotic broken 127 chromosomes was dependent on each other (Figs. 3C and S3C).

129 While the above data suggest that CIP2A acts downstream of MDC1 in promoting the segregation of 130 MDC1-marked broken chromosomes, it also raised a conundrum since loss of MDC1 does not cause 
131 lethality in BRCA2-deficient cells (Fig. S3B). This observation indicates that the MDC1-dependent

132 modulation of DSBs in mitosis is not relevant to the CIP2A-BRCA synthetic lethality.

134 However, TOPBP1 is known to have MDC1-independent roles in promoting genome integrity during

$135 \mathrm{M}$ phase as it also responds to the presence of incompletely replicated DNA that persists until mitosis $136 \quad 26-28$. Analysis of TOPBP1 and CIP2A localization on mitotic chromosomes of wild type or $\mathrm{MDC1}^{-/-}$

137 U2OS cells showed that TOPBP1 and CIP2A colocalized in a number of structures in the absence of

138 IR exposure, and that the frequency of these structures was stimulated by low-dose treatment (400 $139 \mathrm{nM}$ ) with aphidicolin, a DNA polymerase inhibitor (Figs. 3D-F and S3D). The aphidicolin-stimulated 140 structures included small foci that are often symmetrically distributed between the dividing chromatin 141 masses (Fig. 3D, inset $i$ ) as well as filament-like structures that most often occur within the chromatin 142 of the dividing daughter cells, distinguishing them from ultrafine bridges (UFBs) ${ }^{29}$ (Figs. 3E and 143 S3D). We also observed that CIP2A and TOPBP1 localized to centrosomes, a known site of TOPBP1 144 and CIP2A localization ${ }^{30,31}$ (Fig. 3D, inset ii). Centrosomal localization is seen in every cell 145 irrespective of treatment whereas the CIP2A-TOPBP1 foci and filaments were rare in untreated HR146 proficient cells, but their frequency could be increased by aphidicolin treatment in a manner that was 147 mostly independent of MDC1 (Figs. 3F and S3E). Remarkably, in the tumor-derived cell line MDA148 MB-436, which is defective in BRCA1 ${ }^{32}$, CIP2A-TOPBP1 filaments were present in nearly all mitotic 149 cells examined $(91 \pm 2.7 \%, n=3$; Figs. 3G and S3F). In MDA-MB-436 cells, the CIP2A-TOPBP1 150 filaments appear to be seeded from chromosomal loci in mitosis but seem to elongate over time and 151 could sometimes be observed as detached from the chromatin mass in some dividing cells (Fig. S3F).

152 While the nature of these intriguing structures remains under investigation, our data suggest they are 153 initially formed as a consequence of unresolved replication-associated DNA lesions and are thus likely 154 relevant to the $C I P 2 A-B R C A$ synthetic lethality.

156 The intimate and interdependent localization of CIP2A and TOPBP1 on mitotic structures hinted that 157 they may interact with each other. Indeed, CIP2A retrieves TOPBP1 in co-immunoprecipitation 158 assays (Fig. 4A). The two proteins were also found to interact in a cellular co-localization assay where 159 TOPBP1 fused to the LacR DNA-binding domain is targeted to a chromosomal site with 256 copies 160 of the LacO sequence integrated (Fig. 4B-D). The LacR/LacO assay was conducted with interphase 161 cells, suggesting that some CIP2A can shuttle in and out of the nucleus. The TOPBP1-binding region 162 on CIP2A mapped to the highly structured Arm-repeat core (residues 1-560; Fig. 4D), a finding we 163 confirmed by yeast two-hybrid analysis, which also suggested that the interaction is direct (Fig. 4E). 
164 We mapped the CIP2A-interacting region of TOPBP1 to a region located between BRCT5 and 165 BRCT6, in a segment encompassing residues 830-851 (by yeast two-hybrid; Fig. 4e) or residues 776166852 (with LacR/LacO; Figs. 4F and S4AB). Deletion of a segment of TOPBP1 comprising this region, 167 i.e. TOPBP1- $\triangle 756-891$, completely abolished the CIP2A-TOPBP1 interaction as monitored by the $168 \mathrm{LacR} / \mathrm{LacO}$ system or yeast two-hybrid (Fig. 4EF). We then used yeast two-hybrid analysis to identify 169 five point mutants that disrupt binding between CIP2A and TOPBP1 (Fig. S4C), which included 170 mutations that targeted a conserved three-residue segment on TOPBP1, F837-D838-V839 (Fig. 4G). 171 Mutation of these residues to alanine in the context of full-length TOPBP1 generated the TOPBP $1^{3 \mathrm{~A}}$ 172 mutant, which has impaired interaction with CIP2A in both yeast and mammalian cells (Fig. 4EF).

174 The identification of TOPBP1 variants defective in CIP2A binding enabled us to test whether the 175 TOPBP1-CIP2A interaction was essential for the viability of BRCA-deficient cells. We generated 176 DLD1 $B R C A 2^{-/-}$cell lines stably transduced with sgRNA-resistant lentiviruses that express TOPBP1, 177 TOPBP1 ${ }^{3 \mathrm{~A}}$ and TOPBP1- $\Delta 756-891$ (Fig. S4D) and then inactivated the endogenous chromosomal 178 copies of TOPBP 1 by Cas9-mediated mutagenesis. As hinted by the depletion studies, cells expressing 179 TOPBP1- $\triangle 756-891$ and TOPBP $1^{3 \mathrm{~A}}$ failed to form mitotic CIP2A IR-induced foci (Figs. 4H and S4E) 180 and displayed rapid loss of fitness selectively in the $B R C A 2^{-/-}$background upon removal of endogenous 181 TOPBP1 (Figs. 4I and S4F). The lethality of TOPBP1- $\triangle 756-891$ and TOPBP ${ }^{3 \mathrm{~A}}$ in $B R C A 2^{-/}$cells 182 was also accompanied with an increase in micronucleation, suggesting lethal chromosome instability 183 (Figs. 4J and S4G). We conclude that the CIP2A-TOPBP1 interaction is essential for the viability of 184 HR-deficient cells.

\section{Therapeutic proof-of-concept}

187 Our data suggest that disrupting the CIP2A-TOPBP1 interaction may be an attractive therapeutic 188 strategy. To model this approach, we identified a fragment of TOPBP1 corresponding to residues 7561891000 (Fig. 5A), referred to as "B6L" (for BRCT6-long) that is highly effective at disrupting mitotic 190 CIP2A foci when expressed from a lentiviral vector (Figs. 5B and S5A). B6L expression is under the 191 control of a FKBP12-derived destabilization domain (DD) ${ }^{33}$, which enables tight induction of B6L 192 expression upon addition of the Shield-1 or water-soluble AS-1 (Aqua-Shield-1) compounds (Fig. $193 \mathrm{~S} 5 \mathrm{~B})$. Incucyte imaging of $B R C A 2^{-/}$cells following induction of B6L showed a near-complete 194 cessation of proliferation in DLD1 $B R C A 2^{-/-}$cells within 3 days of induction whereas it was innocuous 195 to its BRCA-proficient parental cell line (Fig. 5CD). We also observed that induction of B6L for 2 
days followed by a washout of AS1 (Fig. 5E) led to an irreversible cessation of growth as determined

197 by clonogenic survival (Figs. 5F and S5C) and was accompanied by rapid and high levels

198 micronucleation, further suggesting that segregation of acentric fragments is a plausible cause of cell death in BRCA-deficient cells (Fig. 5G). Disruption of the CIP2A-TOPBP1 interaction with B6L did not impair ATR signaling, ruling out that the impact of B6L is due to ATR misregulation (S5D). B6L expression also caused micronucleation and impaired proliferation of the tumor-derived MDA-MB436 cell line, indicating its ability to stunt proliferation of BRCA-deficient cells and cause chromosome mis-segregation is not limited to engineered backgrounds (Figs. 5G-I and S5EF).

Finally, to test whether disrupting the CIP2A-TOPBP1 interaction could inhibit tumor growth, we established tumors from DLD1 BRCA2-/ cells transduced either with empty (EV) or B6L-expressing lentivirus in NOD-SCID mice. We first characterized the pharmacokinetic properties of the AS-1 compound and found it to be poorly bio-available, but we could optimise a dosing regimen (intraperitoneal injection) that resulted in plasma concentrations that could exceed the EC50 concentration necessary to inhibit growth $B R C A 2^{-/-}$cells for up to $4 \mathrm{hrs}$ per day when administered

211 twice daily (BID dosing; Fig. S6AB). Despite being a suboptimal dosing regimen, the periodic

212 stabilization of B6L in $B R C A 2^{-/-}$tumors was sufficient to cause striking tumor growth inhibition over

213 the course of a 7-day treatment, reaching 85\% (Fig. 5J). Furthermore, tumor growth inhibition was

214 maintained until the completion of the experiment, 8 days after administration of the last dose of AS-

2151 (Fig. 5J). We conclude that not only is the inhibition of the CIP2A-TOPBP1 interaction providing

216 an attractive therapeutic strategy for HR-deficient cancers but our findings indicate that the complete

217 and sustained inhibition of the CIP2A-TOPBP1 interaction may not be necessary for achieving

218 BRCA-deficient tumor control.

\section{Discussion}

221 The observation that acute inactivation of $B R C A 1$ and $B R C A 2$ causes cellular lethality is in line with

222 a model where BRCA1/2-deficient tumors acquire genetic and/or non-genetic adaptive mechanisms

223 that enable these cells to proliferate in the face of HR deficiency. While p53 inactivation is one genetic

224 means by which cells acquire the ability to tolerate HR-deficiency ${ }^{34,35}$, our findings suggest that a 225 dependency on the CIP2A-TOPBP1 complex provides another way to endow HR-deficient cells with 226 the ability to proliferate. Our observations strongly suggest a role for this complex in promoting the 227 segregation of chromosomes that did not fully complete DNA replication (Fig. S6D). We speculate 228 that CIP2A either participates in the resolution of incompletely replicated chromosomes or, more 
229 likely, that it participates in physically bridging acentric fragments to their centromere-bearing

230 counterpart following the mitotic processing of incompletely replicated chromosomes (Fig. 5J). While

231 the molecular details of this function remain to be delineated, we anticipate that this role of CIP2A-

232 TOPBP1 will be distinct from other mitotic DNA damage tolerance pathways ${ }^{29}$ since the genes

233 encoding proteins known to have central roles in these processes, such as RAD52 (MiDAS) or PICH

234 (ultrafine bridge resolution), were not synthetic-lethal with $B R C A 1 / 2$ in either of the CRISPR screens

235 we undertook (Table S1).

237 In conclusion, we nominate the CIP2A-TOPBP1 interaction as a therapeutic target for the treatment 238 of HR-deficient tumors. Since the loss of CIP2A does not cause high loads of DNA damage in HR239 proficient cells, and since Cip2a-deficient mice develop normally with a typical lifespan, we predict 240 that inhibiting the CIP2A-TOPBP1 interaction will have non-overlapping toxicity with PARP 241 inhibitors, and thus could enable a greater range of therapeutic combinations. Furthermore, since we 242 observed that CIP2A loss impairs fitness in a model of PARP inhibitor resistance, $B R C A 1^{-/-} 53 B P 1^{-/-}$ 243 cells (Fig. S6E), inhibitors of the CIP2A-TOPBP1 interaction may also prove effective in subsets of 244 patients that progress on PARP inhibitor therapy. Efforts to discover small molecule inhibitors of the 245 CIP2A-TOPBP1 interaction are currently underway.

\section{Acknowledgments}

248 We thank members of the Durocher lab for helpful discussions and Alain Jeanrenaud for technical 249 help. We also thank Jason Moffat for his generous sharing of the TKO sgRNA libraries. We are 250 grateful to Kin Chan at the NBCC (LTRI) for sequencing. We are also grateful to the Repare in vivo 251 pharmacology team (Anne Roulston, Alexanne Bonneau-Fortin, Marie-Ève Leclaire and Sara 252 Fournier) for their help with tumor xenograft assays. SA was supported by a Banting post-doctoral 253 fellowship and SER was supported by a fellowship from AIRC. AAQ holds and DS held postdoctoral 254 fellowships from the Canadian Institutes for Health Research (CIHR). DD is a Canada Research Chair 255 (Tier I) and work in the DD lab was supported by grants from the CIHR (FDN143343) and Canadian 256 Cancer Society (705644) with additional support from the Krembil Foundation and Repare 257 Therapeutics.

259 Data availability statement

260 All data generated or analysed during this study are included in this published article (and its 261 supplementary information files). 


\section{Code availability}

264 Details about CRISPRcount Analysis (CCA) can be found in the methods, including instructions on

265 how to install the software. Code is also available on github at https://github.com/tohsumi-repare/cca

\section{Conflict of interest statement}

268 DD is a shareholder and advisor of Repare Therapeutics. JD, VB, GM, SYY, RP, JTFY, TO, AV and $269 \mathrm{MZ}$ are employees of Repare Therapeutics. 


\section{References}

2721 Roy, R., Chun, J. \& Powell, S. N. BRCA1 and BRCA2: different roles in a common pathway of genome protection. Nat Rev Cancer 12, 68-78, doi:10.1038/nrc3181

2 Feng, W. \& Jasin, M. BRCA2 suppresses replication stress-induced mitotic and G1 abnormalities through homologous recombination. Nat Commun 8, 525, doi:10.1038/s41467-017-00634-0 (2017).

3 Gowen, L. C., Johnson, B. L., Latour, A. M., Sulik, K. K. \& Koller, B. H. Brca1 deficiency results in early embryonic lethality characterized by neuroepithelial abnormalities. Nat Genet 12, 191-194, doi:10.1038/ng0296-191 (1996).

4 Lord, C. J. \& Ashworth, A. PARP inhibitors: Synthetic lethality in the clinic. Science 355, 1152-1158, doi:10.1126/science.aam7344 (2017).

5 Mengwasser, K. E. et al. Genetic Screens Reveal FEN1 and APEX2 as BRCA2 Synthetic Lethal Targets. Mol Cell, doi:10.1016/j.molcel.2018.12.008 (2019).

6 Alvarez-Quilon, A. et al. Endogenous DNA 3' Blocks Are Vulnerabilities for BRCA1 and BRCA2 Deficiency and Are Reversed by the APE2 Nuclease. Mol Cell 78, 1152-1165 e1158, doi:10.1016/j.molcel.2020.05.021 (2020).

7 Farmer, H. et al. Targeting the DNA repair defect in BRCA mutant cells as a therapeutic strategy. Nature 434, 917-921 (2005).

8 Bryant, H. E. et al. Specific killing of BRCA2-deficient tumours with inhibitors of poly(ADP-ribose) polymerase. Nature 434, 913-917 (2005).

9 Zimmermann, M. et al. CRISPR screens identify genomic ribonucleotides as a source of PARP-trapping lesions. Nature 559, 285-289, doi:10.1038/s41586-018-0291-z (2018).

10 Blessing, C. et al. The Oncogenic Helicase ALC1 Regulates PARP Inhibitor Potency by Trapping PARP2 at DNA Breaks. Mol Cell 80, 862-875 e866, doi:10.1016/j.molcel.2020.10.009 (2020).

11 Mateos-Gomez, P. A. et al. Mammalian polymerase theta promotes alternative NHEJ and suppresses recombination. Nature 518, 254-257, doi:10.1038/nature14157 (2015).

12 Ceccaldi, R. et al. Homologous-recombination-deficient tumours are dependent on Polthetamediated repair. Nature 518, 258-262, doi:10.1038/nature14184 (2015).

13 Dempster, J. M. et al. Extracting Biological Insights from the Project Achilles GenomeScale CRISPR Screens in Cancer Cell Lines. bioRxiv, 720243, doi:10.1101/720243 (2019).

14 Behan, F. M. et al. Prioritization of cancer therapeutic targets using CRISPR-Cas9 screens. Nature, doi:10.1038/s41586-019-1103-9 (2019).

15 Hustedt, N. et al. A consensus set of genetic vulnerabilities to ATR inhibition. Open Biol 9 , 190156, doi:10.1098/rsob.190156 (2019).

16 Kim, E. \& Hart, T. Improved analysis of CRISPR fitness screens and reduced off-target effects with the BAGEL2 gene essentiality classifier. bioRxiv, 2020.2005.2030.125526, doi: $10.1101 / 2020.05 .30 .125526$ (2020).

17 Wang, J. et al. Oncoprotein CIP2A is stabilized via interaction with tumor suppressor PP2A/B56. EMBO Rep 18, 437-450, doi:10.15252/embr.201642788 (2017).

18 Junttila, M. R. et al. CIP2A inhibits PP2A in human malignancies. Cell 130, 51-62, doi:10.1016/j.cell.2007.04.044 (2007).

19 Khanna, A., Pimanda, J. E. \& Westermarck, J. Cancerous inhibitor of protein phosphatase 2A, an emerging human oncoprotein and a potential cancer therapy target. Cancer Res 73, 6548-6553, doi:10.1158/0008-5472.CAN-13-1994 (2013). 
31720 Ventela, S. et al. CIP2A promotes proliferation of spermatogonial progenitor cells and spermatogenesis in mice. PLoS One 7, e33209, doi:10.1371/journal.pone.0033209 (2012). Olivieri, M. et al. A Genetic Map of the Response to DNA Damage in Human Cells. Cell 182, 481-496 e421, doi:10.1016/j.cell.2020.05.040 (2020).

22 Wilson, D. M., 3rd \& Thompson, L. H. Molecular mechanisms of sister-chromatid exchange. Mutat Res 616, 11-23, doi:10.1016/j.mrfmmm.2006.11.017 (2007). Ito, S., Murphy, C. G., Doubrovina, E., Jasin, M. \& Moynahan, M. E. PARP Inhibitors in Clinical Use Induce Genomic Instability in Normal Human Cells. PLoS One 11, e0159341, doi:10.1371/journal.pone.0159341 (2016).

330

331 progression in human cancer cells by regulating the stability and activity of Plk1. Cancer Res 73, 6667-6678, doi:10.1158/0008-5472.CAN-13-0888 (2013).

25 Bunting, S. F. et al. 53BP1 inhibits homologous recombination in Brcal-deficient cells by blocking resection of DNA breaks. Cell 141, 243-254, doi:S0092-8674(10)00285-0 [pii] 10.1016/j.cell.2010.03.012 (2010).

26 Leimbacher, P. A. et al. MDC1 Interacts with TOPBP1 to Maintain Chromosomal Stability during Mitosis. Mol Cell 74, 571-583 e578, doi:10.1016/j.molcel.2019.02.014 (2019).

27 Bagge, J., Oestergaard, V. H. \& Lisby, M. Functions of TopBP1 in preserving genome integrity during mitosis. Semin Cell Dev Biol, doi:10.1016/j.semcdb.2020.08.009 (2020). Broderick, R., Nieminuszczy, J., Blackford, A. N., Winczura, A. \& Niedzwiedz, W. TOPBP1 recruits TOP2A to ultra-fine anaphase bridges to aid in their resolution. Nat Commun 6, 6572, doi:10.1038/ncomms7572 (2015).

29 Ozer, O. \& Hickson, I. D. Pathways for maintenance of telomeres and common fragile sites during DNA replication stress. Open Biol 8, doi:10.1098/rsob.180018 (2018).

30 Bang, S. W. et al. Human TopBP1 localization to the mitotic centrosome mediates mitotic progression. Exp Cell Res 317, 994-1004, doi:10.1016/j.yexcr.2011.01.022 (2011).

31 Jeong, A. L. et al. Cancerous inhibitor of protein phosphatase 2A (CIP2A) protein is involved in centrosome separation through the regulation of NIMA (never in mitosis gene A)-related kinase 2 (NEK2) protein activity. J Biol Chem 289, 28-40, doi:10.1074/jbc.M113.507954 (2014).

32 Elstrodt, F. et al. BRCA1 mutation analysis of 41 human breast cancer cell lines reveals three new deleterious mutants. Cancer Res 66, 41-45, doi:10.1158/0008-5472.CAN-05-2853 (2006).

33 Banaszynski, L. A., Chen, L. C., Maynard-Smith, L. A., Ooi, A. G. \& Wandless, T. J. A rapid, reversible, and tunable method to regulate protein function in living cells using synthetic small molecules. Cell 126, 995-1004, doi:10.1016/j.cell.2006.07.025 (2006).

34 Hakem, R., de la Pompa, J. L., Elia, A., Potter, J. \& Mak, T. W. Partial rescue of Brca1 (5-6) early embryonic lethality by p53 or p21 null mutation. Nat Genet 16, 298-302, doi:10.1038/ng0797-298 (1997).

35 Ludwig, T., Chapman, D. L., Papaioannou, V. E. \& Efstratiadis, A. Targeted mutations of breast cancer susceptibility gene homologs in mice: lethal phenotypes of Brca1, Brca2, Brca1/Brca2, Brca1/p53, and Brca2/p53 nullizygous embryos. Genes \& development 11, 1226-1241 (1997).

36 Noordermeer, S. M. et al. The shieldin complex mediates 53BP1-dependent DNA repair. Nature 560, 117-121, doi:10.1038/s41586-018-0340-7 (2018).

37 Hart, T. et al. High-Resolution CRISPR Screens Reveal Fitness Genes and GenotypeSpecific Cancer Liabilities. Cell 163, 1515-1526, doi:10.1016/j.cell.2015.11.015 (2015). 38 Hart, T. et al. Evaluation and Design of Genome-Wide CRISPR/SpCas9 Knockout Screens. 365 G3 (Bethesda) 7, 2719-2727, doi:10.1534/g3.117.041277 (2017). 
36639 Ohsumi, T. K. \& Borowsky, M. L. MolBioLib: a C++11 framework for rapid development and deployment of bioinformatics tasks. Bioinformatics 28, 2412-2416, doi:10.1093/bioinformatics/bts458 (2012).

40 Hart, T. \& Moffat, J. BAGEL: a computational framework for identifying essential genes from pooled library screens. BMC Bioinformatics 17, 164, doi:10.1186/s12859-016-1015-8 (2016).

41 Delignette-Muller, M. L. \& Dutang, C. fitdistrplus: An R Package for Fitting Distributions. 2015 64, 34, doi:10.18637/jss.v064.i04 (2015).

42 Tang, J. et al. Acetylation limits 53BP1 association with damaged chromatin to promote homologous recombination. Nature structural \& molecular biology, doi:10.1038/nsmb. 2499 (2013). 


\section{METHODS}

381 Cell culture

382 RPE1-hTERT, U2OS and $293 \mathrm{~T}$ cells were grown at $37^{\circ} \mathrm{C}$ and $5 \% \mathrm{CO}_{2}$ in DMEM supplemented with 383 10\% FBS (Wisent \#080150) and 1\% Pen/Strep (Wisent). Parental and BRCA2 ${ }^{-/-}$DLD1 cells were 384 purchased from Horizon and maintained in RPMI-1640 medium (ATCC 30-2001) supplemented with $38510 \%$ FBS and 1\% Pen/Strep. Parental and BRCA2 $2^{-/-}$DLD1 Cas9 cells were generated through viral 386 infection with lentiCas9-Blast (Addgene \#52962) followed by blasticidin selection. MDA-MB-436 387 cells were purchased from ATCC and maintained in DMEM supplemented with 10\% FBS and 1\% 388 Pen/Strep. DLD1 and MDA-MB-436 cell lines were grown at $37^{\circ} \mathrm{C}$ in a low-oxygen $\left(3 \% \mathrm{O}_{2}\right)$

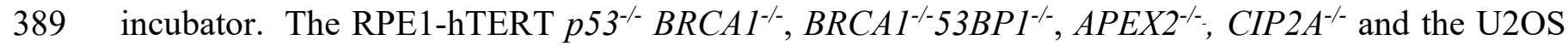
$390 \mathrm{MDCl}^{-/-}$knockout cell lines were described previously $6,15,26,36$. The $C I P 2 A^{-/-}$RPE1-hTERT cell line 391 (i.e. p53 $^{+}$) is described in de Marco Zompit et al. (submitted).

\section{Lentiviral transduction}

394 Lentiviral particles were produced in $293 \mathrm{~T}$ cells in 10-cm plates by co-transfection of $10 \mu \mathrm{g}$ of 395 targeting vector with $3 \mu \mathrm{g}$ VSV-G, $5 \mu \mathrm{g}$ pMDLg/RRE and $2.5 \mu \mathrm{g}$ pRSV-REV (Addgene \#14888, 396 \#12251, \#12253) using calcium phosphate. Viral transductions were performed in the presence of 4 $397 \mu \mathrm{g} / \mu \mathrm{L}$ polybrene (Sigma-Aldrich) at a multiplicity of infection (MOI) $<1$. Transduced cells were 398 selected by culturing in the presence of blasticidin (InvivoGen) or nourseothricin (Jena Bioscience) 399 depending on the lentiviral vector used.

\section{Two-color competitive growth assays}

402 Cells were transduced with sgRNA expression lentiviruses, either expressing NLS-mCherry-sgAAVS1 403 (control) or an NLS-GFP-sgRNA targeting a specific gene of interest (Supplementary Table 3) at an 404 MOI of 0.5. $24 \mathrm{~h}$ after transduction, cells were selected for $48 \mathrm{~h}$ using $15 \mu \mathrm{g} / \mathrm{mL}$ (RPE1) or $2 \mu \mathrm{g} / \mathrm{mL}$ 405 (DLD1) puromycin (Life Technologies). $96 \mathrm{~h}$ after transduction, mCherry- and GFP-expressing cells 
were mixed 1:1 (2,000 cells each for RPE1; 3,000 cells each for RPE1 BRCA1 ${ }^{-/}$and DLD1; 9,000 cells each for DLD1 $B R C A 2^{-/}$) and seeded in a 12-well plate. Cells were imaged for GFP and mCherry $24 \mathrm{~h}$ after initial plating $(\mathrm{t}=0)$ and at the indicated timepoints using a $4 \mathrm{X}$ objective InCell Analyzer system (GE Healthcare Life Sciences, Marlborough). Segmentation and counting of GFP- and mCherry-positive cells were performed using an Acapella script (PerkinElmer, Waltham). Efficiency of indel formation was analysed by performing PCR amplification of the region surrounding the sgRNA sequence using DNA isolated from cells collected from 4 to 7 days after transduction and subsequent ICE analysis (https://ice.synthego.com/\#/) (Supplementary Table 4).

\section{Clonogenic survival assays}

416 Cells were transduced at low MOI $(<1.0)$ with lentivirus derived from pLentiGuide (RPE1 cells) or

417 pLentiCRISPRv2, which expressed sgRNAs targeting CIP2A, TOPBP1 or AAVS1 (which was used 418 as control). Puromycin-containing medium was added the next day to select for transductants and cells 419 were seeded for clonal growth $48 \mathrm{~h}$ later. Cells were seeded in 10-cm dishes (750-5,000 cells per 10 $420 \mathrm{~cm}$ plate, depending on cell line and genotype). For drug sensitivity assays, cells were seeded into 421 media containing a range of camptothecin (Sigma) concentrations (for determination of camptothecin 422 sensitivity) or in regular media after several days of AS1 treatment (i.e. after induction of B6L). For 423 clonogenic survival assays performed with $C I P 2 A^{-/-}$cells, plates were incubated in atmospheric 424 oxygen. Experiments performed with $B R C A 1^{-/-}$and $B R C A 2^{-/}$cells and their controls were incubated 425 at 3\% $\mathrm{O}_{2}$. Medium was refreshed after $7 \mathrm{~d}$. After 14-20 d, colonies were stained with a crystal violet 426 solution $(0.4 \%(\mathrm{w} / \mathrm{v})$ crystal violet (Sigma), 20\% methanol). Colonies were manually counted or 427 counted using a GelCount instrument (Oxford Optronix). Data were plotted as surviving fractions 428 relative to untreated cells or $s g A A V S 1$-transduced controls.

\section{$430 \quad$ Plasmids}

431 For CRISPR-Cas9 genome editing, sgRNAs were cloned either in lentiCRISPRv2 or in lentiguide432 NLS-GFP as in ref ${ }^{6}$. The sgRNA sequences used in this study are included in Supplementary Table 433 3. The pcDNA5-FRT/TO-LacR-FLAG-TOPBP1 plasmid was obtained from Addgene (\#31313). 434 Point mutants were introduced by site-directed mutagenesis using Quikchange (Agilent). For TOPBP1 435 rescue experiments, the pLenti-CMVie-IRES-BlastR (pCIB) plasmid was obtained from Addgene 
436 (\#119863). pCIB-2xHA was generated by cloning a double HA tag with a flanking NotI site in pCIB, 437 using AscI and BamHI restriction sites. The TOPBPI coding sequence was amplified from pcDNA5438 FRT/TO-LacR-FLAG-TopBP1 and mutagenised at Genscript (Piscataway, NJ) to generate an 439 sgRNA-resistant construct with a silent mutation at Thr263 (ACC to ACA). This fragment was then 440 cloned into pCIB-2xHA using NotI and BamHI restriction sites to generate pCIB-2xHA-TOPBP1$441 \mathrm{sgR}$. For the inducible expression of the B6L fragment, we first synthesized a cassette coding the 442 FKBP-derived destabilization domain (DD) ${ }^{33}$ along with an EcoRI restriction site and a single FLAG 443 tag (Genscript). This cassette was then cloned into pHIV-NAT-T2A-hCD52 (kind gift of R. Scully) 444 using the NotI and BamHI restriction sites to generate pHIV-NAT-DD-FLAG. pHIV-NAT-DD445 FLAG-B6L was amplified by PCR from pcDNA5-FRT/TO-LacR-FLAG-TOPBP1(756-1000) and 446 cloned into pHIV-NAT-DD-FLAG using EcoRI and BamHI sites. The CIP2A coding sequence was 447 amplified from a BirA-CIP2A expression plasmid (a kind gift from A.-C. Gingras) and cloned into 448 the pcDNA5-FRT/TO-FLAG vector using the AscI and EcoRI sites. The mutation making this vector 449 resistant to $\operatorname{sgCIP} 2 A-2$ (silent mutation in Ala650, GCC to GCA) was introduced by site-directed 450 mutagenesis generating pcDNA5-FRT/TO-Flag-CIP2A-sg2R. Using this vector as a template, FLAG451 CIP2A or portions of CIP2A were amplified by PCR and cloned into the pHIV-NAT-T2A-hCD52 452 using NotI and EcoRI restriction sites. The corresponding control vector, pHIV-NAT-FLAG-T2A453 hCD52, and pHIV-NAT-FLAG-CIP2A(560-915) were generated by deletion PCR from pHIV-NAT454 FLAG-CIP2A-sg2R. For yeast two hybrid experiments, a fragment corresponding to CIP2A (1-560) 455 was cloned by Genscript into pGADT7 AD (Clontech/Takara) to create a fusion with the GAL4 456 activating domain using EcoRI and XmaI restriction sites, whereas a TOPBP1 fragment 457 corresponding to residues 2-1523 was amplified from pCDNA5-FRT/TO-LacR-FLAG-TopBP1 and 458 cloned into pGBKT7 (Clontech/Takara) to create a fusion with the GAL4 DNA binding domain using 459 the NdeI and XmaI sites. pGBKT7-GAL4-BD-TOPBP1- $4756-891$ and pGBKT7-GAL4-BD460 TOPBP1-3A were derived from pGBKT7-GAL4-BD-TOPBP1, removing the sequence coding 461 residues 756-891 by deletion PCR and mutating the codons for Phe837, Asp838, Val839 to Ala by 462 Quikchange site-directed mutagenesis, respectively. pGBKT7-GAL4-BD-TOPBP1(830-851) was 463 generated by cloning a TOPBP1 fragment corresponding to residues 830-851 into pGBKT7 using the 464 NdeI and XmaI restriction sites. The alanine scanning library of the TOPBP1 830-851 fragment was 465 generated at Genscript and cloned into pGBKT7-GAL4-BD. 
468 The CRISPR screens were carried out using protocols derived from refs ${ }^{6,9,37}$. Synthetic lethality

469 screens are basically undertaken as two parallel screens with a parental cell line and an isogenic variant

470 with one genetic alteration, in this case $B R C A 1$ or $B R C A 2$ loss-of-function mutations. For the BRCA2

471 screen, DLD1 parental and BRCA2-/- cells were transduced with the lentiviral TKOv3 sgRNA library

$472 \quad 37,38$ at a low MOI ( 0.3) and media containing puromycin (Life Technologies) was added the next

473 day to select for transductants. The following day, cells were trypsinized and replated in the same

474 plates while maintaining puromycin selection. $3 \mathrm{~d}$ after infection, which was considered the initial

475 time point (t0), cells were pooled together and divided into 2 sets of technical replicates. Cells were

476 grown for a period of 18-30 d and cell pellets were collected every $3 \mathrm{~d}$. Each screen was performed as

477 a technical duplicate with a theoretical library coverage of $\geq 400$ cells per sgRNA maintained at every

478 step. Genomic DNA was isolated using the QIAamp Blood Maxi Kit (Qiagen) and genome-integrated

479 sgRNA sequences were amplified by PCR using NEBNext Ultra II Q5 Master Mix (New England

480 Biolabs). i5 and i7 multiplexing barcodes were added in a second round of PCR and final gel-purified

481 products were sequenced on an Illumina NextSeq500 system at the LTRI NBCC facility

482 (https://nbcc.lunenfeld.ca/) to determine sgRNA representation in each sample.

484 CRISPRCount Analysis (CCA)

485 CCA is a scoring approach optimized for isogenic CRISPR screens that provides gene-level scores

486 and ranking of genes according to the impact of their targeting sgRNAs between test and control 487 samples. CCA also aims to prioritize sgRNAs that are selectively deleterious to fitness in the test 488 samples. CCA is available on Docker. To download the Docker image of CCA, install Docker 489 (https://www.docker.com/) and then in a terminal window, execute: "docker pull tohsumirepare/cca".

490 The CCA Docker image source is located at https://github.com/tohsumi-repare/cca and the 491 documentation for CCA, such the input file format and method of execution, is in the doc folder.

492 CCA employs non-parametric statistics. Implementation of CCA was based on MolBioLib ${ }^{39}$ 493 (sourceforge.net/projects/molbiolib) and includes the Mann-Whitney U test from ALGLIB C++ 494 (www.alglib.net). The input of CCA is a matrix of samples versus sgRNAs where the entries are the 495 sgRNA readcounts in that sample. The CCA score is computed as follows: (1) normalization of the 496 readcount file so that each sample's count over all sgRNAs is 10 million; (2) removal of sgRNAs with 497 readcounts at $\mathrm{T} 0$ is $<30$ to avoid false positives due to low readcounts; (3) computing a depletion 
matrix of samples versus sgRNAs where the depletion $=1-($ count at final time $) /($ count at initial time $)$

$499=1-$ foldchange. The depletion is such that it is maximum, 1 , if the test sample has no viable cells at

500 the final timepoint. The depletion may be negative if there is proliferation of cells at the final

501 timepoint. By default, we limit the minimum value of the depletion of all control samples to 0 (doing

502 otherwise can create false positive hits if $\operatorname{sgRNAs}$ cause proliferation in control samples).

503 For a given gene, we let the vector of depletion values over all test samples be denoted t and over all 504 control samples be denoted c. For vector v, let Q3(v) be the third quantile of v. The CCA score for 505 that gene is:

Score $=\left\{\mathrm{A}^{*}\right.$ median $\left.(\mathrm{t})+\mathrm{B}^{*} \mathrm{Q} 3(\mathrm{t})+\mathrm{C}^{*}(\operatorname{median}(\mathrm{t})-\operatorname{median}(\mathrm{c}))+\mathrm{D}^{*}(\mathrm{Q} 3(\mathrm{t})-\mathrm{Q} 3(\mathrm{c}))\right\} *$ $\left\{1-(\text { likelihood } \mathrm{t}<\text { non-essential })^{\mathrm{E}}\right\} *\left\{1-(\text { likelihood } \mathrm{c}>\text { essential })^{\mathrm{F}}\right\} *$

where $\mathrm{A} \cong 2, \mathrm{~B} \cong 0.017, \mathrm{C} \cong 0.02, \mathrm{D}=1, \mathrm{E} \cong 8.8, \mathrm{~F} \cong 0.35, \mathrm{G} \cong 7.1$, and $\mathrm{H} \cong 0.22$. Likelihoods are computed using Mann-Whitney $U$ test where the inequality is tested by taking either the right or left tail and the equality is tested by taking both tails. For comparison with essential and non-essential 513 genes, we use the gene sets described in ${ }^{40}$ (github.com/hart-lab/bagel). For essential genes, we use 514 depletion values of all samples of all sgRNAs associated with an essential gene. For isogenic screens, we subtract 10,000 from all genes whose median(t) is less than zero. The top 3000 CCA scores are modeled using a beta distribution fitted using the fitdistrplus package ${ }^{41}$ in $\mathrm{R}$. Taking the top genes with $\mathrm{p}<0.05$, we stratify them into 4 Jenks classes using the classInt package in R (cran.rproject.org/web/packages/classInt/index.html). The values of the parameters, A through H except D, 519 were determined by using a derivative-free optimiziation method, BiteOpt

520 (github.com/avaneev/biteopt), to minimize:

$$
=1 /\left(\begin{array}{c}
\frac{1}{4} * \text { numInTop } 300+\text { numInTop } 200+2 * \text { numInTop } 100+ \\
4 * \text { numInTop } 50+8 * \text { numInTop } 25+16 * \text { numInTop } 10+32 * \text { numInTop } 5+1
\end{array}\right)
$$

522 where numInTopN is the number of positive control synthetic lethal genes found in the top $\mathrm{N}$ genes 523 as ranked by CCA's scoring method over all training screens that have positive controls. D is always 
527 set to 1 , as the other variables, A, B, and C, may be scaled. For the purpose of screens presented in

528 this work, we considered a gene a hit if it is present in the top two Jenks classes.

530 Public Cancer Dependency Data

531 Cell line panel estimates of gene dependency based on CRISPR screens were used in the analysis.

532 CERES scores were downloaded from the 2020 Q1 release of the Broad Cancer Dependency Map

533 (https://depmap.org/portal/download/). Copy Number Bias Corrected Fold Change Values were

534 downloaded from the April 2019 release of the Sanger Project Score

535 (https://score.depmap.sanger.ac.uk/downloads). The following cell lines were classified as

536 BRCA1/2 biallelic mutants: COV362_OVARY, DOTC24510_CERVIX, HCC1395_BREAST,

537 HCC1599_BREAST, ICC15_BILIARY_TRACT, JHOS2_OVARY, JHOS4_OVARY,

538 MDAMB436_BREAST, SUM149PT_BREAST, CAPAN1_PANCREAS,

539 SUM1315MO2_BREAST and UWB1289_OVARY. See Supplementary Table 4 for values.

$541 \quad$ Antibodies

542 The antibodies listed below were used for immunoblotting (IB) or immunofluorescence (IF). Primary 543 antibodies: mouse anti-CIP2A (clone 2G10-3B5; Santa Cruz sc80659, 1:500 IF, 1:1000 IB), rabbit 544 anti-CIP2A (Cell Signalling Technologies \#14805, 1:5000-12000 IB), rabbit anti-phospho-Histone 545 H2A.X (Ser139) (Cell Signalling Technologies \#2577, 1:500 IF), mouse anti-phospho-Histone 546 H2A.X (Ser139) (clone JBW301; Millipore Sigma \#05-636, 1:5000 IF), mouse anti-CHK1 (Santa 547 Cruz sc8408, 1:500 IB), rabbit anti-phospho-CHK1 (Ser345) (Cell Signalling Technologies \#2348, 548 1:1000 IB), rabbit anti-KAP1 (Bethyl A300-274A, 1:10000 IB), HRP-conjugated mouse anti-FLAG 549 M2 (Sigma A8592, 1:1000-5000 IB), mouse anti-FLAG M2 (Sigma G1804, IB 1:1000), rat anti550 FLAG (BioLegend \#637301, 1:1000 IF), rabbit anti-TOPBP1 (Abcam ab2402, 1:2000 IF, 1:5000 IB 551 or 1:1500 IB using yeast extracts), rabbit anti-TOPBP1 (ABE1463, Millipore, 1:300 IF), mouse anti552 alpha-tubulin (Calbiochem CP06, 1:2000 IB), rat anti-HA (Roche 11867423001, 1:200 IB and IF or 553 1:500 IB using yeast extracts), mouse anti-CENPA (Abcam ab13939, 1:2000 IF), sheep anti-MDC1 554 (Serotec/Bio-Rad AHP799, 1:1000 IF), rabbit anti-MDC1 (Abcam ab11171, 1:1000 IF), rabbit anti- 
555 GAL4 DNA Binding Domain (Upstate 08283, 1:5000 IB), rat anti-Tubulin (YOL1/34) (Abcam 556 ab6161, 1:2000 IB).

557 Secondary antibodies for immunoblots: IRDye 800CW goat anti-mouse IgG and IRDye 680RD goat 558 anti-rabbit IgG (LiCOR 926-32210 and 926-68071, 1:5000 or 1:50000 using yeast extracts), HRP-

559 conjugated sheep anti-mouse IgG (GE Healthcare NA931, 1:5000), HRP-conjugated goat anti-rabbit

560 IgG (Cedarlane \#111-035-144, 1:5000), and HRP-conjugated goat anti-rat IgG (Cedarlane 112-035-

$561003,1: 5000$ or 1:50000 using yeast extracts). Secondary antibodies for immunofluorescence:

562 AlexaFluor 488-donkey anti-rat IgG (Thermo Fisher Scientific A21208, 1:2000), AlexaFluor 647-

563 donkey anti-mouse IgG (Thermo Fisher Scientific A31571, 1:2000), AlexaFluor 488-goat anti-mouse

564 IgG (Thermo Fisher Scientific A11029, 1:2000 or 1:1000 for high content microscopy), AlexaFluor 565 555-goat anti-mouse IgG (Thermo Fisher Scientific A21424, 1:2000), AlexaFluor 647-goat anti566 mouse IgG (ThermoFisher Scientific A21236, 1:2000), AlexaFluor 647-goat anti-rabbit IgG

567 (ThermoFisher Scientific A21244, 1:2000 IF), AlexaFluor 488-goat anti-rabbit IgG (Thermo Fisher 568 Scientific A11034, 1:2000), AlexaFluor 555-donkey anti-sheep IgG (Thermo Fisher Scientific 569 A21436, 1:2000), AlexaFluor 568-goat anti-rabbit IgG (Thermo Fisher Scientific A11011, 1:1000).

\section{Short interfering RNAs}

572 The following siRNAs were used in this study: Dharmacon siGENOME Non-Targeting siRNA \#3 D573 001210-03-20, ON-TARGET Plus KIAA1524 (CIP2A) SMARTpool L-014135-01-0005, 574 siGENOME MDC1 SMARTpool M-003506-04-0005, siGENOME TOPBP1 SMARTpool M575 012358-01-0005).

\section{Fine chemicals}

578 The following drugs were used in the course of the study: camptothecin (CPT, Sigma-Millipore), 579 hydroxyurea (Sigma), nocodazole (Sigma), Shield-1 (Takara Bio USA, Inc), Aqua-Shield-1 (AS1;

580 CheminPharma) and aphidicolin (Focus Biochemicals, 10-2058). Concentration and duration of 581 treatment is indicated in the legends of the corresponding figures. 


\section{High content imaging}

584 To analyze $\gamma \mathrm{H} 2 \mathrm{AX}$ focus formation, cells were seeded in 96 -well plates $(\sim 7,500$ cells/well $)$, cultured 585 for $24 \mathrm{~h}$, incubated in medium containing $20 \mathrm{mM}$ EdU (5-ethynyl-2-deoxyuridine, Life Technologies) 586 for the final 30 min and then washed with PBS and fixed with 4\% paraformaldehyde (PFA) in PBS 587 for $10 \mathrm{~min}$ at room temperature (RT). Cells were then processed for $\gamma \mathrm{H} 2 \mathrm{AX}$ staining. Prior to the click 588 reaction, immunocomplexes were fixed again using 4\% PFA/PBS for $5 \mathrm{~min}$. Cells were rinsed with 589 PBS and incubated with EdU staining buffer $(150 \mathrm{mM}$ Tris/HCl pH 8.8, $1 \mathrm{mM} \mathrm{CuSO} 4,100 \mathrm{mM}$ 590 ascorbic acid and $10 \mathrm{mM}$ AlexaFluor 647 Azide (Life Technologies)) for $30 \mathrm{~min}$. After rinsing with 591 PBS, images were acquired on an IN Cell Analyzer 6000 automated microscope (GE Life Sciences) 592 with a $60 \mathrm{X}$ objective. Image analysis was performed using Columbus (PerkinElmer). Cell cycle 593 profiling and analysis was evaluated based on EdU and DAPI staining.

\section{Immunofluorescence}

596 Cells were grown and fixed on glass coverslips with 2-4\% PFA, permeabilized with $0.3 \%$ Triton X597100 in PBS, and blocked with 5\% BSA in PBS + 0.2\% Tween-20. Cells were then stained for $2 \mathrm{~h}$ with 598 primary antibodies in blocking buffer, washed three times with PBS $+0.2 \%$ Tween-20, incubated for $5991 \mathrm{~h}$ with appropriate secondary antibodies plus $0.8 \mathrm{ug} / \mathrm{ml} \mathrm{DAPI}$, then washed twice with PBS $+0.2 \%$

600 Tween-20 and a final wash with PBS. Coverslips were mounted onto glass slides with ProLong Gold 601 mounting reagent (Invitrogen). Images were acquired using a Zeiss LSM780 laser-scanning 602 microscope (Oberkochen, Germany). Foci were manually counted.

603 For assessing the colocalization of MDC1, TOPBP1 and CIP2A, U2OS cells were reverse transfected 604 with a final concentration of 10nM siRNA using Lipofectamine RNAiMAX (Invitrogen) on coverslips 605 in 6-well plates. Nocodazole was added to the media at a final concentration of $100 \mathrm{ng} / \mathrm{mL} 16 \mathrm{~h}$ before 606 collection. $48 \mathrm{~h}$ after transfection, cells were irradiated with 2 Gy of ionizing radiation using a Faxitron 607 X-ray cabinet (Faxitron, Tucson, AZ) and allowed to recover for $1 \mathrm{~h}$ prior to fixation as described for 608 immunofluorescence. Foci were counted manually and at least 50 mitotic cells per condition were 609 imaged in each experiment. 
610 For the experiments relating to the mitotic structures labelled by CIP2A and TOPBP1, U2OS wild-

611 type and $\mathrm{MDC1}^{-/-}$cell lines were seeded on coverslips and either treated with $400 \mathrm{nM}$ aphidicolin for

$61216 \mathrm{~h}$ (overnight) or left untreated. In order to perform immunofluorescence, cells were quickly washed

613 once with cold PBS and then fixed with ice-cold methanol for 10 min on ice. Methanol was discarded

614 and cells were washed two times with PBS before incubation with blocking buffer (10\% FBS in PBS)

615 for at least $1 \mathrm{~h}$. Incubation with primary antibodies diluted in 5\% FBS-PBS was performed overnight

616 at $4^{\circ} \mathrm{C}$ in a humidity chamber. Coverslips were then washed $3 \times 10$ min with blocking buffer and

617 incubated with AlexaFluor-conjugated secondary antibodies for $1 \mathrm{~h}$ at room temperature in the dark.

618 After washing $3 \times 10$ min with PBS, coverslips were mounted on glass microscopy slides (Thermo

619 Scientific, 630-1985, dimensions L76 X W26 mm) with VECTASHIELD mounting medium

620 containing $0.5 \mu \mathrm{g} / \mathrm{mL}$ (DAPI) (Vector Laboratories, H-1200).

621 Confocal images were acquired using a Leica SP8 inverse confocal laser scanning microscope with a 622 63x, 1.4-NA Plan-Apochromat oil-immersion objective. The sequential scanning mode was applied, 623 and the number of overexposed pixels was kept at a minimum. Images were recorded using optimal 624 pixel size based on Nyquist criterion. At least 10 fields per condition with 10 to $15 \mathrm{z}$-sections were 625 acquired, with 8-bit depth. Quantification of the foci was performed manually based on maximum 626 intensity projections. Representative grayscale images were pseudocolored and adjusted for 627 brightness and contrast in Adobe Photoshop CC 2020 by using adjustment layers.

\section{Immunoblotting}

630 Cell pellets were boiled for 5-10 min in 2X SDS sample buffer (20\% (v/v) glycerol, 2\% (w/v) SDS, $6310.01 \%(\mathrm{w} / \mathrm{v})$ bromophenol blue, $167 \mathrm{mM}$ Tris-Cl pH 6.8, $20 \mathrm{mM} \mathrm{DTT})$ and separated by SDS-PAGE 632 on gradient gels (Invitrogen). Proteins were transferred to nitrocellulose membranes (GE Healthcare), 633 then blocked with 5\% FBS or 5\% milk in TBST and probed for $2 \mathrm{~h}$ with primary antibodies. 634 Membranes were washed three times for $5 \mathrm{~min}$ with TBST, then probed with appropriate secondary 635 antibodies for $1 \mathrm{~h}$, and washed again with TBST, three times for $5 \mathrm{~min}$. Secondary antibody detection 636 was achieved using an Odyssey Scanner (LiCOR) or enhanced chemiluminescence (ECL, Thermo 637 Fisher Scientific \#34579). 


\section{Cytogenetic analyses}

640 To monitor chromosome aberrations, $0.5 \times 10^{6}$ puromycin-selected RPE1-hTERT cells of the 641 indicated genotypes were seeded in 10-cm dishes $3 \mathrm{~d}$ after transduction with virus particles expressing 642 NLS-GFP-sgAAVS1 (control) or an NLS-GFP-sgRNA targeting a specific gene of interest. $4 \mathrm{~d}$ later $643100 \mathrm{ng} / \mathrm{mL}$ KaryoMAX colcemid (Gibco/Thermo Fisher) was added for $2 \mathrm{~h}$, and cells were harvested. 644 To analyze sister chromatid exchange, $0.75 \times 10^{6}$ RPE1-hTERT cells of the indicated genotypes were 645 seeded in 10-cm dishes. $24 \mathrm{~h}$ after seeding, $\mathrm{BrdU}$ (final concentration $10 \mu \mathrm{M}$ ) was added to the media 646 and cells were grown for 48 h; 100 ng/mL KaryoMAX colcemid (Gibco/Thermo Fisher) was added 647 for the final $2 \mathrm{~h}$. For cell harvesting, growth medium was stored in a conical tube. Cells were gently 648 washed and treated twice for $5 \mathrm{~min}$ with $1 \mathrm{~mL}$ of trypsin. The growth medium and the $2 \mathrm{~mL}$ of 649 trypsinization incubations were centrifuged (1000 rpm, $\left.5 \mathrm{~min}, 4^{\circ} \mathrm{C}\right)$. Cells were then washed with PBS 650 and resuspended in $75 \mathrm{mM} \mathrm{KCl}$ for $15 \mathrm{~min}$ at $37^{\circ} \mathrm{C}$. Cells were centrifuged again, the supernatant was 651 removed and cells were fixed by drop-wise addition of $1 \mathrm{~mL}$ fixative (ice-cold methanol:acetic acid, 652 3:1) while gently vortexing. An additional $9 \mathrm{~mL}$ of fixative was then added, and cells were incubated 653 at $4^{\circ} \mathrm{C}$ for at least $16 \mathrm{~h}$. Once fixed, metaphases were dropped on glass slides and air-dried overnight, 654 protected from light.

655 To visualize chromosomal aberrations, slides were dehydrated in a 70\%, 95\% and 100\% ethanol series 656 (5 min each), air-dried and mounted in DAPI-containing ProLong Gold mounting medium (Molecular 657 Probes/Thermo Fisher). To visualize sister chromatid exchanges (SCE) slides were rehydrated in PBS 658 for $5 \mathrm{~min}$ and stained with $2 \mu \mathrm{g} / \mathrm{mL}$ Hoechst 33342 (Thermo Fisher) in 2xSSC (final concentration $659300 \mathrm{mM} \mathrm{NaCl}, 30 \mathrm{mM}$ sodium citrate, $\mathrm{pH}$ 7.0) for $15 \mathrm{~min}$. Stained slides were placed in a plastic tray, 660 covered with a thin layer of $2 x S S C$ and irradiated with $254 \mathrm{nM} \mathrm{UV} \mathrm{light}\left(\sim 5400 \mathrm{~J} / \mathrm{m}^{2}\right)$. Slides were 661 subsequently dehydrated in a 70\%,95\% and 100\% ethanol series (5 min each), air-dried and mounted 662 in DAPI-containing ProLong Gold mounting medium (Molecular Probes/Thermo Fisher). Images 663 were captured on a Zeiss LSM780 laser-scanning confocal microscope.

666 For monitoring recruitment of endogenous CIP2A to FLAG-tagged TOPBP1 foci we used U2OS667 FokI cells, which contain an integrated LacO array. These cells, which are known also as U2OS- 
$668 \mathrm{DSB}^{42}$, are referred to in the text as $\mathrm{U} 2 \mathrm{OS}-\mathrm{lacO}_{256}$ cells because we used them without any induction

669 of FokI. $1.8 \times 10^{5}$ cells were seeded in 6-well plates containing glass coverslips. $24 \mathrm{~h}$ after seeding, 670 cells were transfected using $1 \mu \mathrm{g}$ of pcDNA5-LacR-FLAG or pcDNA5-LacR-FLAG-TopBP1 (full 671 length, fragments, or mutants) using Lipofectamine 2000. Cells were fixed with 4\% PFA 48 h after 672 transfection and stained for immunofluorescence. For monitoring recruitment of Flag-CIP2A, U2OS673 FokI cells were transduced with pHIV-NAT constructs. After $0.1 \mathrm{mg} / \mathrm{mL}$ nourseothricin selection and 674 cell expansion, $2 \times 10^{5}$ cells were seeded in 6-well plates. The next day, cells were transfected using $6751 \mu \mathrm{g}$ of pcDNA5-LacR-TOPBP1. $24 \mathrm{~h}$ later, cells were seeded in a 96-well plate $(\sim 20,000$ cells per 676 well), cultured for $24 \mathrm{~h}$ and fixed with 2\% PFA and stained for immunofluorescence. Images were 677 acquired on an IN Cell Analyzer 6000 automated microscope (GE Life Sciences) with a 60X 678 objective.

\section{Cell proliferation (IncuCyte) assays}

681 MDA-MB-436, DLD1 wild-type and DLD1 $B R C A 2^{-/-}$cells were infected with an empty virus 682 containing the destabilization domain (DD) alone (pHIV-NAT-DD-FLAG) or virus containing an 683 expression cassette for DD-tagged B6L (pHIV-NAT-DD-FLAG-TOPBP1-756-891). After 684 nourseothricin selection $(0.1 \mathrm{mg} / \mathrm{mL}$ for MDA-MB-436, $0.2 \mathrm{mg} / \mathrm{mL}$ for DLD1) and cell expansion, 685 cells were seeded in 96-well plates (500-4,000 cells depending on cell line and genotype) and treated 686 with $1 \mu \mathrm{M}$ of Shield-1 or Aqua-Shield-1. The following day, plates were transferred into an IncuCyte 687 Live-Cell Analysis Imager (Essen/Sartorius). Cell confluency was monitored every $4 \mathrm{~h}$ up to $10 \mathrm{~d}$ 688 post-seeding.

691 For TOPBP1 rescue experiments, DLD1 wild-type and $B R C A 2^{-/-}$cells stably expressing 2xHA692 TOPBP1 were generated by viral transduction and selection with blasticidin $(7.5 \mu \mathrm{g} / \mathrm{mL}$ for parental 693 cells, $10 \mu \mathrm{g} / \mathrm{mL}$ for $B R C A 2^{-/-}$cells). $3 \mathrm{~d}$ after transduction with sgRNA viral particules (as described 694 in the clonogenic survival assays), cells were seeded in a 96-well plate (1,500 for wild-type cells; 6954,000 for $B R C A 2^{--}$cells) and cultured for 4 additional days. For inducible B6L expression 696 experiments, DLD1 and MDA-MB-436 cells were seeded in a 96-well plate (1,500 for DLD1 wild- 
type cells; 4,000 for DLD1 BRCA2-/- cells; 14,000 for MDA-MB-436) and cultured for up to 4 days

698 in the presence of Aqua-Shield-1. For detection of micronuclei, cells were fixed with 2\% PFA, washed

6993 times with PBS, permeabilized with 0.3\% Triton X-100 in PBS for 5 min, washed 3 times with PBS,

700 incubated for $1 \mathrm{~h}$ with PBS + DAPI $(0.5 \mu \mathrm{g} / \mathrm{mL})$. Alternatively, cells were stained for

701 immunofluorescence (CENPA detection). After the last wash with PBS, images were acquired on an

702 IN Cell Analyzer 6000 automated microscope (GE Life Sciences) with a 40X objective. Micronuclei were automatically detected and counted using the Columbus analysis tool (PerkinElmer).

\section{Yeast assays}

706

Yeast two-hybrid assay was conducted using Matchmaker GAL4 two-hybrid system 3

(Clontech/Takara, USA). Bait and prey vectors were co-transformed into the yeast strain AH109

(Clontech/Takara, USA), using a standard high-efficiency transformation protocol, and plated onto media lacking tryptophan and leucine (SD-Trp-Leu) for $3 \mathrm{~d}$ to select for cells harboring the two plasmids. Single colonies were isolated and the interaction between bait and prey was assessed by a serial deletion assay based on the ability to grow on selective media lacking leucine, tryptophan,

712 histidine and adenine (SD-Leu-Trp-His-Ade). Viability assays were performed using yeast cultures

713 grown overnight at $30^{\circ} \mathrm{C}$ in SD-Trp-Leu to maintain plasmid selection. Ten-fold serial dilutions of 714 cells were spotted on SD-Trp-Leu and SD-Leu-Trp-His-Ade containing 5 mM 3-amino-1,2,4-triazole 715 (3-AT). Plates were imaged after $4 \mathrm{~d}$ of incubation at $30^{\circ} \mathrm{C}$.

\section{Yeast protein extracts}

For protein extracts, the cellular pellet of $20 \mathrm{~mL}$ of cell suspension $\left(1 \times 10^{7}\right.$ cells $\left./ \mathrm{mL}\right)$ was washed twice with $1 \mathrm{~mL}$ of $20 \%$ trichloroacetic acid (TCA) and suspended in $50 \mu \mathrm{L}$ of $20 \%$ TCA. Cells were broken with acid-washed glass beads (Sigma G8772) by vortexing for 3 minutes at maximum speed. After addition of $100 \mu \mathrm{L}$ of $5 \%$ TCA, precipitated proteins were transferred into a new $1.5 \mathrm{~mL}$ tube and centrifuged at $3000 \mathrm{rpm}$ for $10 \mathrm{~min}$ at room temperature. The supernatant was removed, and the pellets of proteins suspended in $100 \mu \mathrm{L}$ of 2 X SDS sample buffer $(20 \%$ (v/v) glycerol, $2 \%(\mathrm{w} / \mathrm{v})$ SDS, $0.01 \%$

724 (w/v) bromophenol blue, $167 \mathrm{mM}$ Tris-Cl pH 6.8, $20 \mathrm{mM}$ DTT)). The pH was neutralized with $60 \mu 1$ 725 of $2 \mathrm{M}$ Tris base. The protein extract was boiled for 5 minutes at $95^{\circ} \mathrm{C}$ and centrifuged for 2 minutes 726 at top speed at room temperature. The supernatant was collected, and the protein extract was subjected to SDS-PAGE analysis. 


\section{Co-immunoprecipitation studies}

729 Confluent $293 \mathrm{~T}$ cells, either untreated or treated with $100 \mathrm{ng} / \mathrm{mL}$ nocodazole (Sigma) for $16 \mathrm{~h}$, were 730 used for each co-immunoprecipitation experiment. Cells were scraped directly into PBS, pelleted by

731 centrifugation at $1000 \mathrm{x} g$ for 5 minutes, and lysed by incubation in lysis buffer $(50 \mathrm{mM}$ Tris- $\mathrm{HCl} \mathrm{pH}$ $7328,100 \mathrm{mM} \mathrm{NaCl}, 2 \mathrm{mM}$ EDTA, $10 \mathrm{mM} \mathrm{NaF}, 0.5 \%$ NP-40, $10 \mathrm{mM} \mathrm{MgCl}$, 1x cOmplete EDTA-free 733 Mini EDTA-free protease inhibitor tablet (Sigma), 1x Phosphatase inhibitor cocktail 3 (Sigma) and 5 $734 \mathrm{U} / \mathrm{mL}$ benzonase (Sigma)) for $30 \mathrm{~min}$ on ice. Lysates were then cleared by centrifugation at 21,000 $\mathrm{x}$ $735 g$ for $10 \mathrm{~min} .1 \mu \mathrm{g}$ of either mouse anti-CIP2A (2G10-3B5; Santa Cruz sc-80659) or normal mouse 736 IgG (EMD Millipore 12-371) were added to the lysate and incubated with rotation at $4^{\circ} \mathrm{C}$ for $1 \mathrm{~h}$. 737 Subsequently, $20 \mu \mathrm{L}$ of a slurry of protein G Dynabeads (Invitrogen) were added to the lysates and 738 incubated for an additional $1 \mathrm{~h}$ at $4^{\circ} \mathrm{C}$. Beads were collected using a magnetic rack and washed $4 \times 5$ 739 min with $500 \mu \mathrm{L}$ lysis buffer, then boiled in $25 \mu \mathrm{L} 2 \mathrm{X}$ SDS sample buffer. The presence of co740 immunoprecipitated proteins were detected by immunoblotting.

\section{Pharmacokinetic measurements}

742 Whole blood was collected in over a period of $8 \mathrm{hr}$ from conscious mice by tail snip and

743 volumetrically transferred to tubes containing $0.1 \mathrm{M}$ citrate (3:1 ratio blood/citrate) and frozen ($74480^{\circ} \mathrm{C}$ ). The determination of the total blood concentration was performed by protein precipitation 745 extraction, followed by reversed-phase liquid chromatography and electrospray mass spectrometry

746 (LC-MS/MS). Blood concentration versus time data was converted to plasma concentrations using

747 an in vitro measurement of the blood to plasma ratio. The data were expressed as free plasma

748 concentration using the fraction unbound which was assessed by equilibrium dialysis of AS-1 in

749 mouse plasma over a period of 6 hours. PK profiles over a 24-hour period were estimated using

750 Phoenix WinNonlin 8.3.1.

752 Animals

753 Experiments were conducted in female NOD-SCID (Nonobese diabetic/Severe combined 754 immunodeficiency) mice (5-7 weeks old, Charles River, St. Constant, Canada). Mice were group755 housed on autoclaved corncob bedding in individual HEPA ventilated cages (Innocage ${ }^{\circledR}$ IVC, 756 Innovive, San Diego, CA, USA $)$ in a temperature-controlled environment $\left(22 \pm 1.5^{\circ} \mathrm{C}, 30-80 \%\right.$ relative 757 humidity, 12-h light/dark). Mice were acclimatized in the animal facility for at least $5 \mathrm{~d}$ prior to use. 758 Studies were conducted under a protocol that has been approved by Animal Care Committee. Animals 
759 were housed and experiments were performed at the Neomed site (Montreal, Canada), which has

760 accreditation from CCAC (Canadian Council on Animal Care). Experiments were performed during

761 the light phase of the cycle. Animals had irradiated food (Harlan Teklad, Montreal, Canada) and filtered

762 water ad libitum. The number of animals used was the minimum necessary to achieve an $80 \%$

763 statistical power to detect a $40 \%$ change.

\section{Cancer cell implantation and measurement}

766 DLD1 $\mathrm{BRCA}^{-/} \mathrm{EV}$ and B6L-expressing cells were harvested during exponential growth and re767 suspended with high glucose RPMI1640 media (\#30-2001, ATCC). Mice received a subcutaneous 768 (SC) injection of $10 \times 10^{6}$ DLD1 $B R C A 2^{-/-}$cells EV or B6L-expressing cells, in a volume of $0.1 \mu 1$, into

769 the right flank. Tumor volume (TV) and body weight (BW) were measured 2-3 times per week. When 770 tumors reached the target size of $150-200 \mathrm{~mm}^{3}$ mice were randomized into several groups $(\mathrm{n}=8)$ and 771 treatment with AS-1 was initiated. Randomization was done to establish similar tumor volume mean 772 and standard deviation in each group. AS-1 was administered Intraperitoneal (IP) twice daily (BID) in 773 a volume of $5 \mathrm{ml} / \mathrm{kg}$ in phosphate buffered saline (PBS). TV were measured using a digital caliper and 774 calculated using the formula $0.52 \times \mathrm{L} \times \mathrm{W}^{2}$. Response to treatment was evaluated for tumor growth 775 inhibition (\%TGI). TGI was defined as the formula: \% TGI $=\left(\left(\mathrm{TV}_{\text {vehicle/last }}-\mathrm{TV}_{\text {vehicle/day } 0)}\right)\left(\mathrm{TV}_{\text {treated/last }}-\right.\right.$

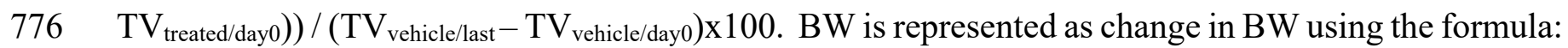
$777 \% \mathrm{BW}$ change $=\left(\mathrm{BW}_{\text {last }} / \mathrm{BW}\right.$ day $) \times 100$. Statistical significance relative to vehicle control was 778 established by two-tailed unpaired Student's t-test (Excel); ${ }^{*} \mathrm{p}<0.05 ;{ }^{*} \mathrm{p}<0.01 ; * * * \mathrm{p}<0.001$. All data 779 are presented as the mean \pm standard error of the mean. 


\section{FIGURE 1}

A

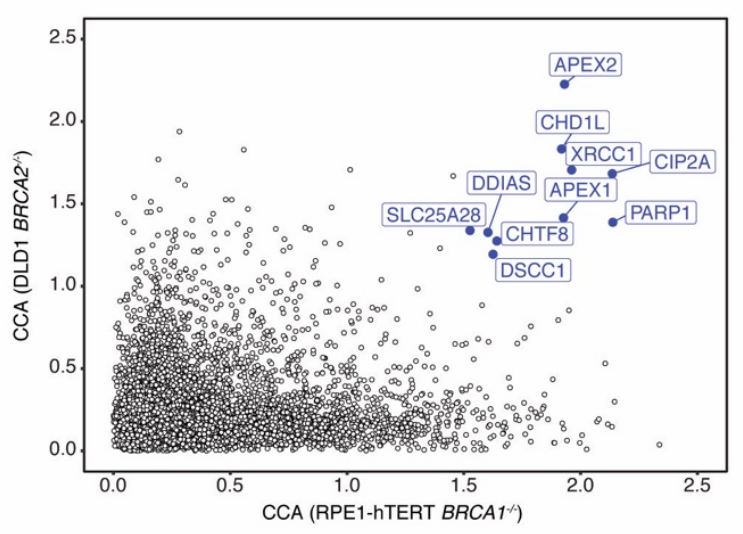

C

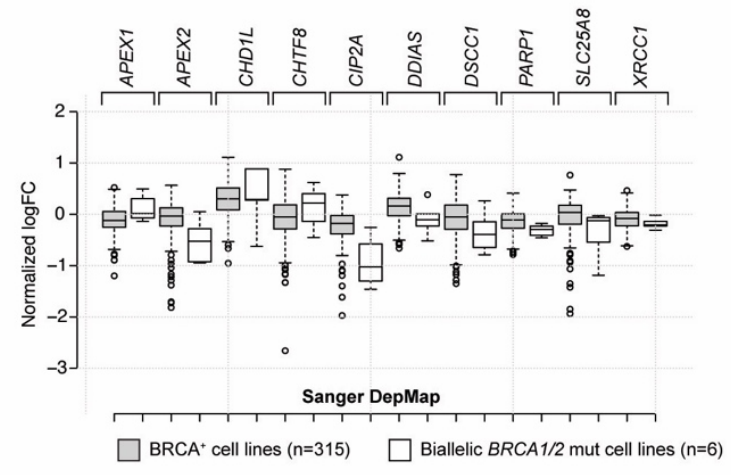

$\mathbf{F}$

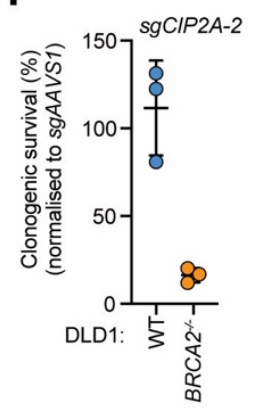

G DLD1 BRCA2-
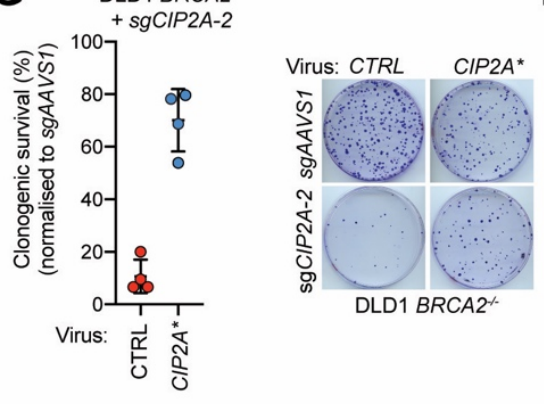

I

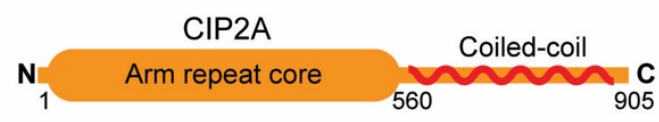

B
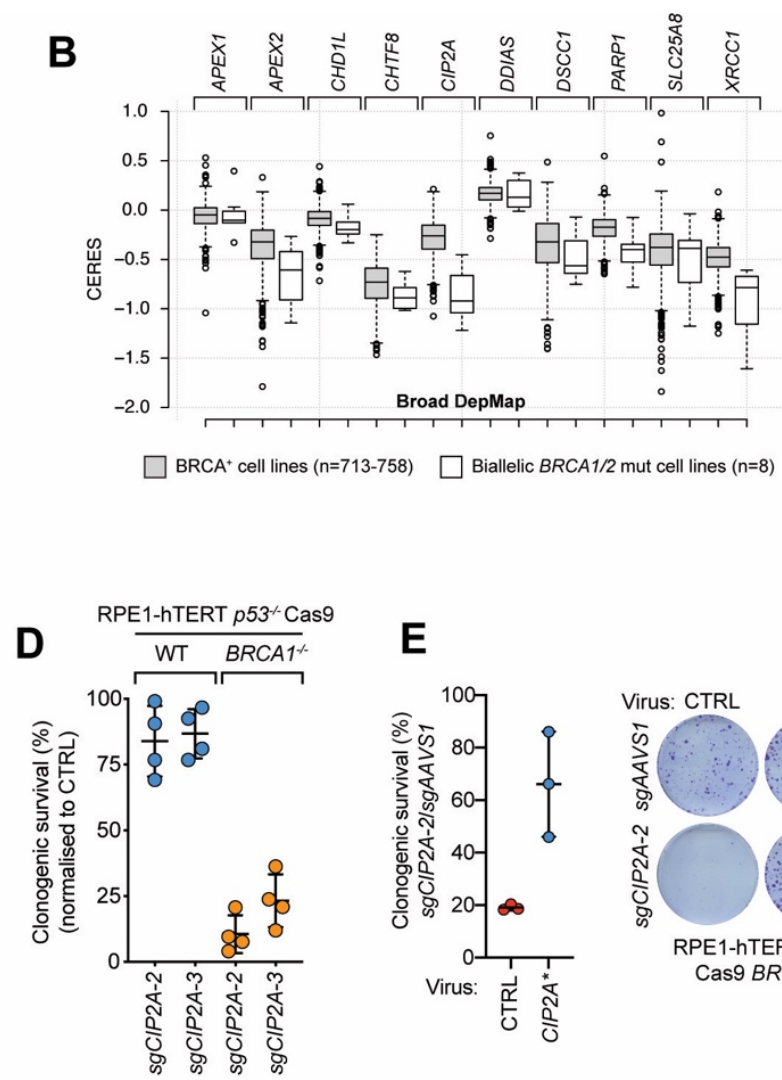

E
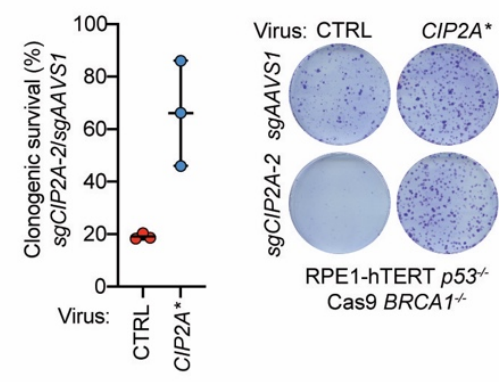

$\mathbf{H}$

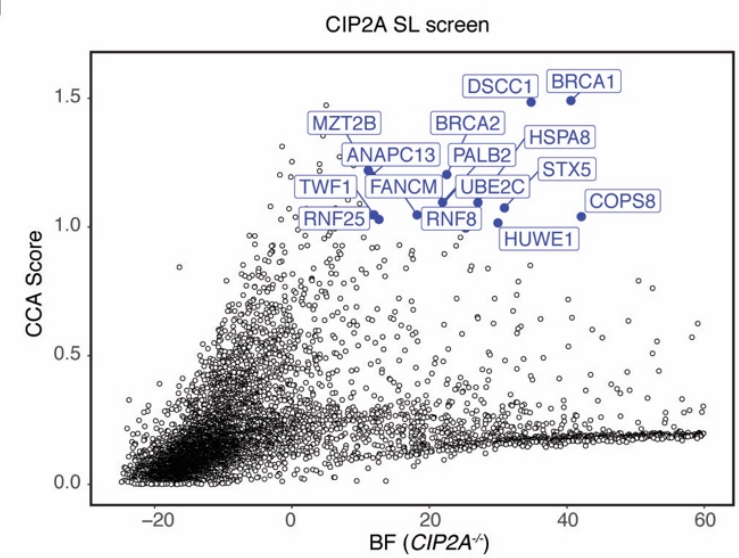

Fig. 1. CIP2A loss is synthetic-lethal with $B R C A 1$ - or $B R C A 2-d e f i c i e n c y$. (A) Scatter plot of CCA scores for the CRISPR synthetic lethality screens in $B R C A 1^{-/}$and $B R C A 2^{-/-}$cells. Highlighted in blue are the top 10 genes common to both screens. $(\mathbf{B}, \mathbf{C})$ Boxplots of essentiality scores for the indicated genes derived from the Broad (B) and Sanger (C) DepMap projects. Cell lines were grouped according to whether or not they harbored biallelic inactivating mutations in $B R C A 1$ or $B R C A 2$. Center lines show the medians; box limits indicate the 25th and 75th percentiles; whiskers extend 1.5 times the interquartile range, outliers are represented by dots. See Fig. S1B for statistical analysis of the results. (D) Clonogenic survival of RPE1-hTERT $p 53^{-/-}$Cas9 wild-type (WT) and $B R C A 1^{-/-}$cells expressing 
the indicated $C I P 2 A$-targeting sgRNAs or transduced with control lentivirus (CTRL: either empty virus or virus with sgRNA targeting $A A V S 1)$. Data was normalized to the plating efficiency of the control virus. Data are shown as mean \pm S.D. $(\mathrm{n}=4)$. (E) Reintroduction of a sgRNA-resistant $C I P 2 A$ transgene $\left(\mathrm{CIP} 2 \mathrm{~A}^{*}\right.$ ) rescues lethality of RPE1-hTERT $p 53^{-/-}$Cas9 $B R C A 1^{-/-}$cells caused by $\operatorname{sgCIP} 2 A$ 2. Data are shown as mean \pm S.D. $(\mathrm{n}=3)$. (F) Clonogenic survival of DLD1 wild-type (WT) and $B R C A 2^{-/-}$cells expressing $C I P 2 A$ - or $A A V S 1$-targeting sgRNAs. Data was normalized to the plating efficiency of cells expressing $s g A A V S 1$. Data are shown as mean \pm S.D. $(\mathrm{n}=4)$. (G) Reintroduction of a sgRNA-resistant CIP $2 A$ transgene rescues lethality in DLD1 $B R C A 2^{--}$cells caused by $s g C I P 2 A-2$. Data are shown as mean \pm S.D. $(\mathrm{n}=3)$. (H) Scatter plot of CCA scores (y-axis) and Bayes Factor (BF) values derived from BAGEL2 (x-axis, for $C I P 2 A^{-/}$cell line) for the $C I P 2 A$ isogenic synthetic lethal screen. (I) Schematic representation of the CIP2A protein. 


\section{FIGURE 2}

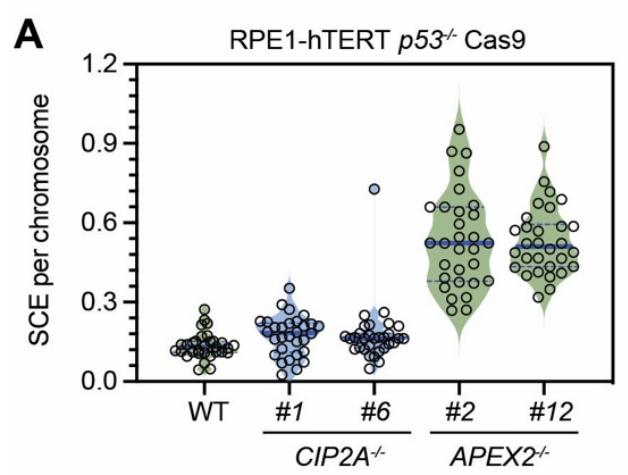

D

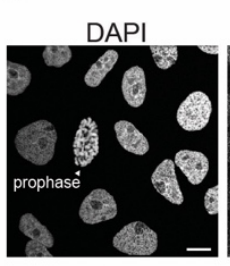

B

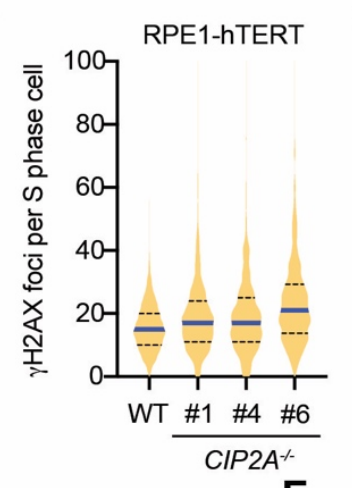

C Interphase DLD1 cells

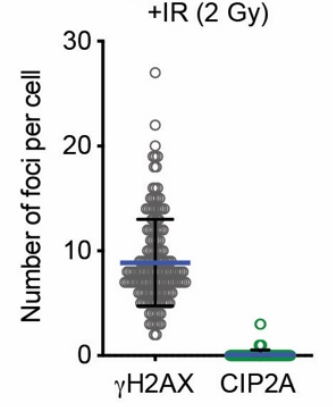

E Mitotic DLD1 cells

F
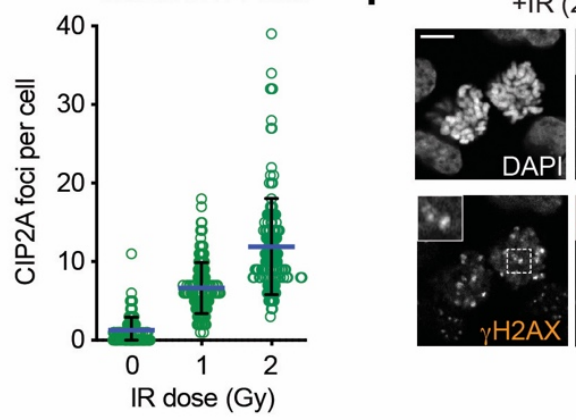

$\mathrm{DLD} 1(\mathrm{WT})$
$+\operatorname{IR}(2 \mathrm{~Gy})$

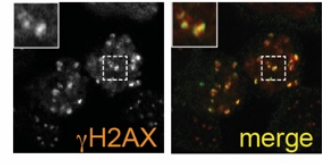

G

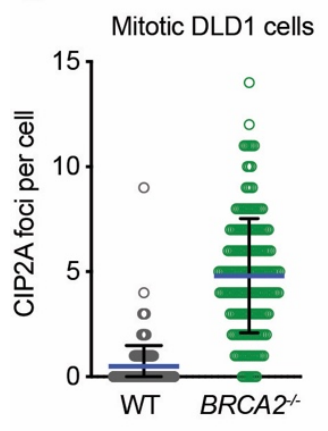

H

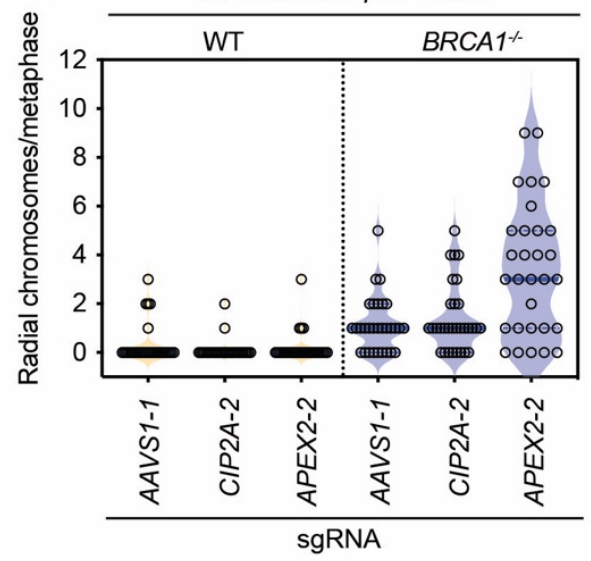

I

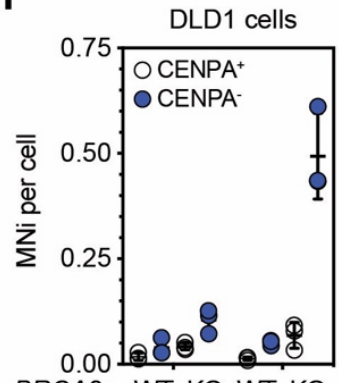

BRCA2: WT KO WT KO

sgRNA: $A A V S 1$ CIP2A-2

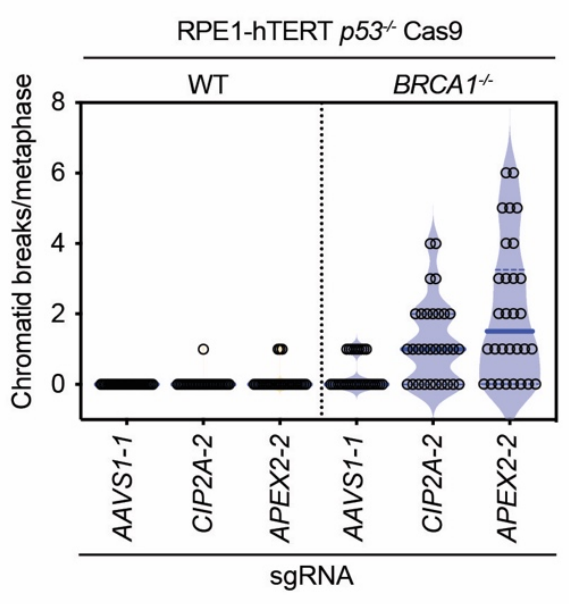


Fig. 2. CIP2A prevents acentric chromosome segregation. (A) Analysis of spontaneous sister chromatid exchanges (SCEs) in RPE1-hTERT p53/- Cas9-derived cell lines of the indicated genotype. The violin plot summarizes data from 3 biological replicates. The blue line is the median and dashed lines are at $1^{\text {st }}$ and $3^{\text {rd }}$ quartiles. (B) Violin plot of the automated quantitation of $\gamma \mathrm{H} 2 \mathrm{AX}$ foci in $\mathrm{S}$ phase cells of RPE1-hTERT wild-type (WT) and indicated CIP $2 A^{-/-}$clones. The blue line is at the median and $25^{\text {th }}$ and $75^{\text {th }}$ quartiles are indicated with dashed lines; $\mathrm{n}=8361$ (WT), 3891 (CIP2A $\left.A^{-/} \# 1\right), 2869$ (CIP2 $A^{-/-\# 4), ~} 1924$ (CIP2 $A^{-/-\# 6) . ~(C) ~ Q u a n t i t a t i o n ~ o f ~} \gamma \mathrm{H} 2 \mathrm{AX}$ and CIP2A IR-induced foci, $1 \mathrm{~h}$ postIR (2 Gy) in interphase DLD1 cells. Plot represents the aggregate of 3 independent experiments. The bar is at the median \pm S.D. (D) Representative micrographs of the experiment shown in C. (E) Quantitation of $\gamma \mathrm{H} 2 \mathrm{AX}$ and CIP2A IR-induced foci, $1 \mathrm{~h}$ post-IR (2 Gy) in mitotic DLD1 cells. Plot represents aggregate of 3 independent experiments. The bar is at the median \pm S.D. (F) Representative micrographs of the experiment shown in (e). Scale bar $=10 \mu \mathrm{m}$. (G) Quantitation of spontaneous CIP2A foci in mitotic DLD1 parental (WT) or $B R C A 2^{-/-}$cells. Plot represents aggregate of 3 independent experiments. The bar is at the median \pm S.D. (H) Quantitation of radial chromosomes (left) and chromatid breaks (right) in metaphase spreads from RPE1-hTERT $p 53^{-/}$Cas9 cells upon transduction of virus expressing sgRNAs targeting AAVS1, APEX2 or CIP2A (10 metaphases scored from at least 2 biologically independent experiments). Representative images are shown in Fig. S2E. (I) Quantitation of micronuclei (MNi) staining positive (+) or negative (-) for CENPA in DLD1 cells, parental (WT) or $B R C A 2^{-/}(\mathrm{KO}), 7 \mathrm{~d}$ post-transduction with indicated sgRNAs. Biological replicates are shown and the bars represent the mean \pm S.D. Representative micrographs are shown on the right. Arrowheads point at micronuclei. Scale bar $=10 \mu \mathrm{m}$. 


\section{FIGURE 3}

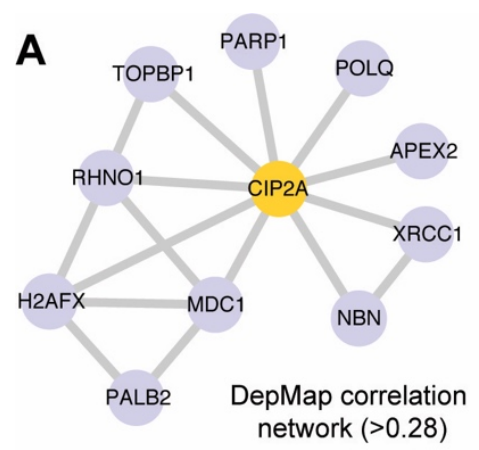

B
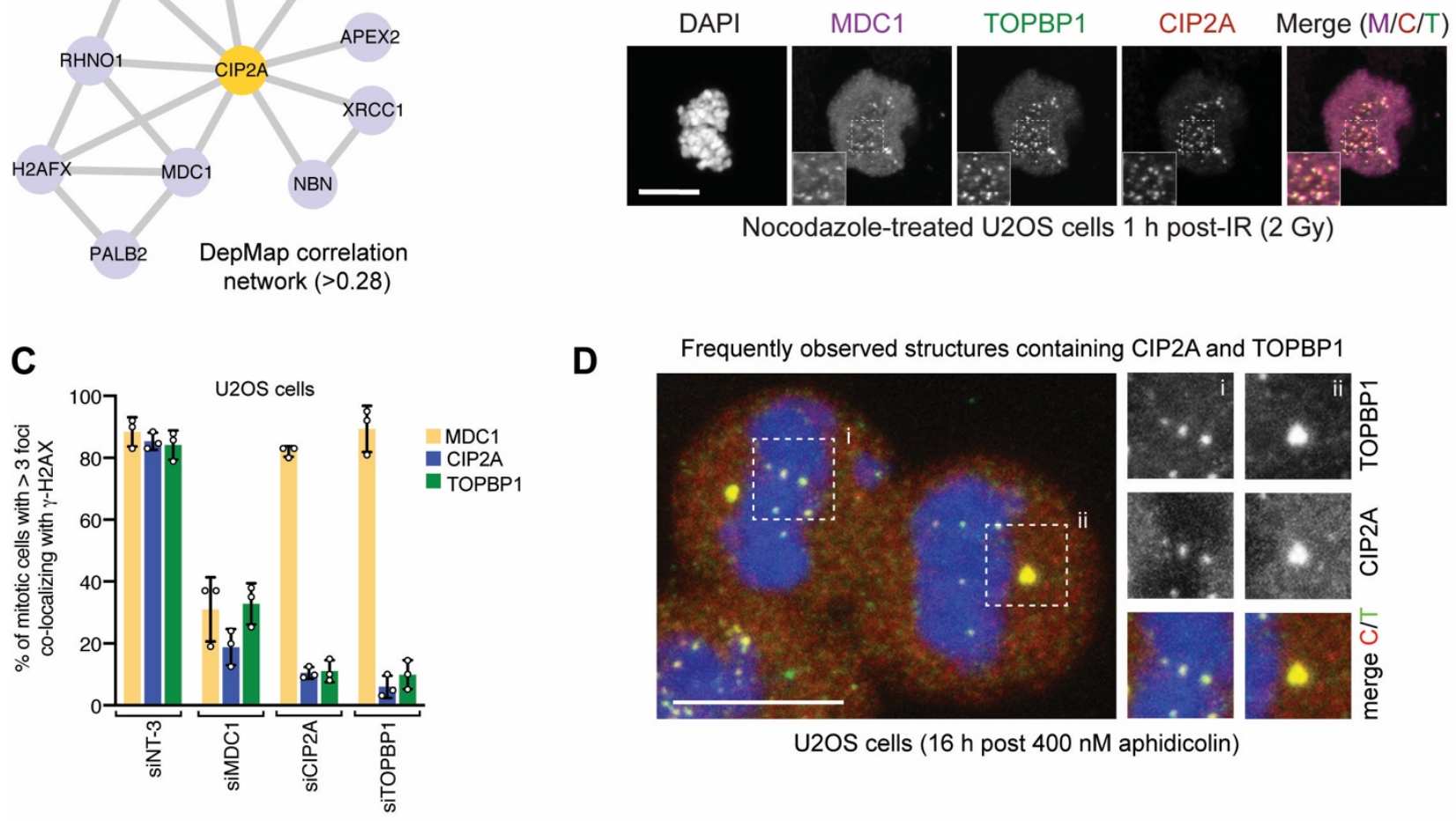

D

Frequently observed structures containing CIP2A and TOPBP1

E

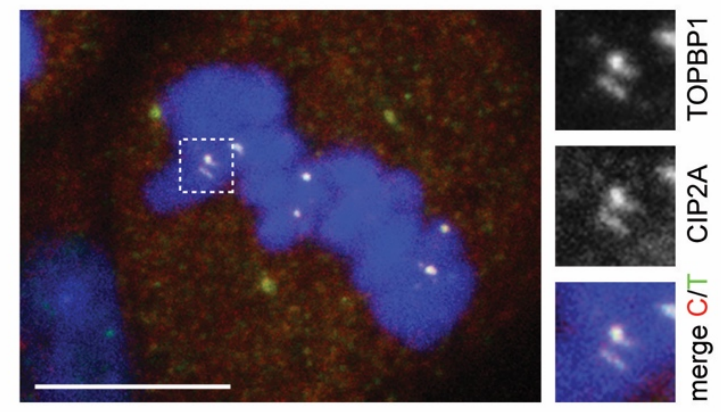

U2OS cells ( $16 \mathrm{~h}$ post $400 \mathrm{nM}$ aphidicolin)
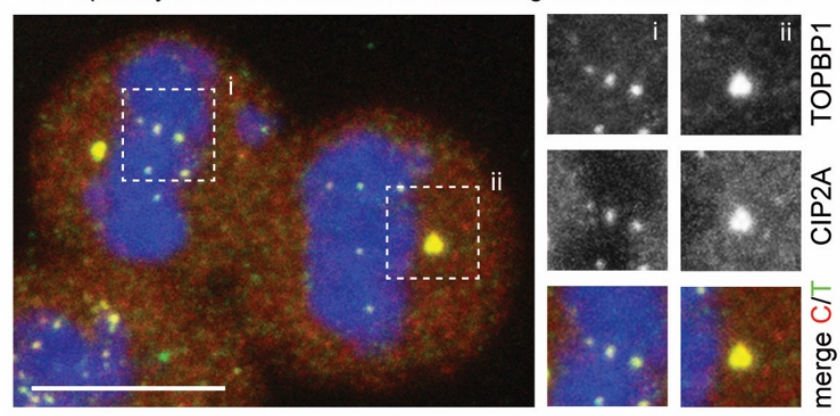

U2OS cells (16 h post $400 \mathrm{nM}$ aphidicolin)

$\mathbf{F}$

G

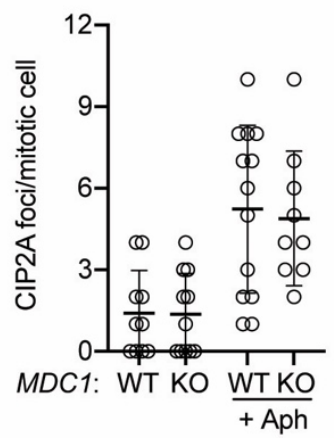

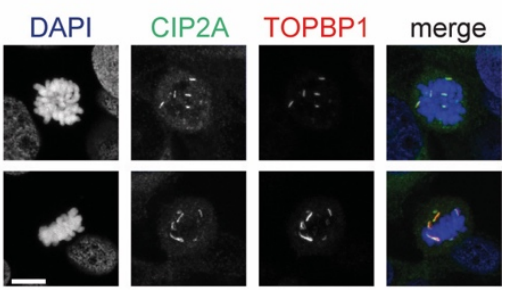

MDA-MB-436

Mitotic nuclei with filaments: $91+/-2.7 \%, n=3$

Fig. 3. CIP2A co-localizes with TOPBP1 on mitotic structures. (A) Correlation network based on Pearson correlation of gene-level dependency scores $(>0.28)$ derived from the Broad DepMap data. (B) Representative micrograph of an X-irradiated (2 Gy) mitotic U2OS cell treated with $100 \mathrm{ng} / \mathrm{mL}$ nocodozole for $16 \mathrm{~h}$ and stained with the indicated antibodies. DNA was stained with DAPI. Scale bar $=10 \mu \mathrm{m}$. (C) Quantitation of MDC1, CIP2A and TOPBP1 IR-induced foci in mitotic U2OS cells treated with nocodazole and the indicated siRNAs (siNT-3 is a non-targeting control). Data is presented as the mean \pm S.D. $(n=3)$. Representative micrographs are shown in Fig. S3B. (D, E) Types of CIP2A/TOPBP1 structures observed in mitotic cells after treatment with low dose aphidicolin. Maximum intensity projections of confocal z-stacks of U2OS wild type mitotic cells treated with 400 $\mathrm{nM}$ aphidicolin for $16 \mathrm{~h}$. Scale bars $=10 \mu \mathrm{m}$. Besides centrosomes (D, inset $i i)$ that always stain for TOPBP1 and CIP2A regardless of the treatment, small round foci are the most frequently observed 
structures in response to aphidicolin treatment (D, inset $i$ ). Less frequently observed structures include curved and straight filamentous assemblies (E and Fig. S3D). (F) Quantitation of CIP2A and TOPBP1 colocalizing foci in U2OS (WT) and $\mathrm{MDCl}^{-/-}(\mathrm{KO})$ cells after treatment with $400 \mathrm{nM}$ aphidicolin (16 h). The number of foci per mitotic cell are shown and the bars represent the mean \pm S.D. (G) Representative micrographs of MDA-MB-436 mitotic cells stained for CIP2A and TOPBP1. DNA was stained with DAPI. Scale bars $=10 \mu \mathrm{m}$. Quantitation of the percentage of cells with filaments is indicated. 
bioRxiv preprint doi: https://doi.org/10.1101/2021.02.08.430060; this version posted February 8, 2021. The copyright holder for this preprint (which was not certified by peer review) is the author/funder, who has granted bioRxiv a license to display the preprint in perpetuity. It is made available under aCC-BY-NC-ND 4.0 International license.

FIGURE 4

A

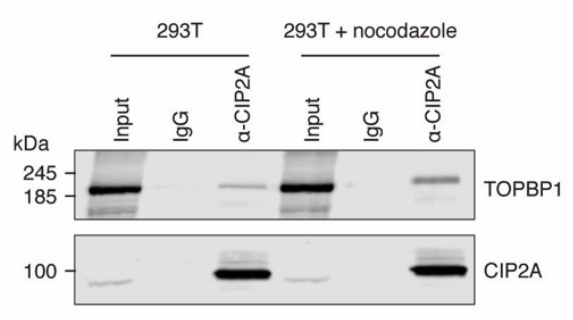

B LacRlaco interaction assay

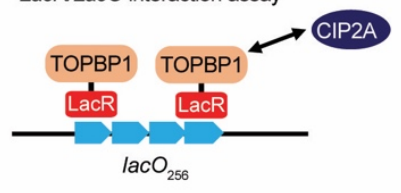

$\mathbf{E}$

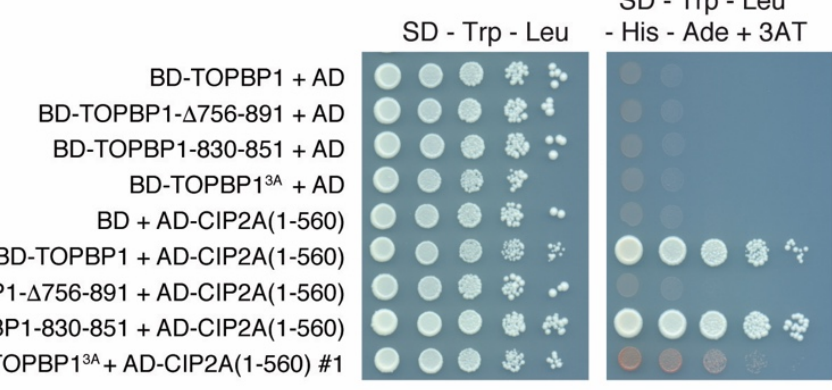

4 days, $30^{\circ} \mathrm{C}$
G

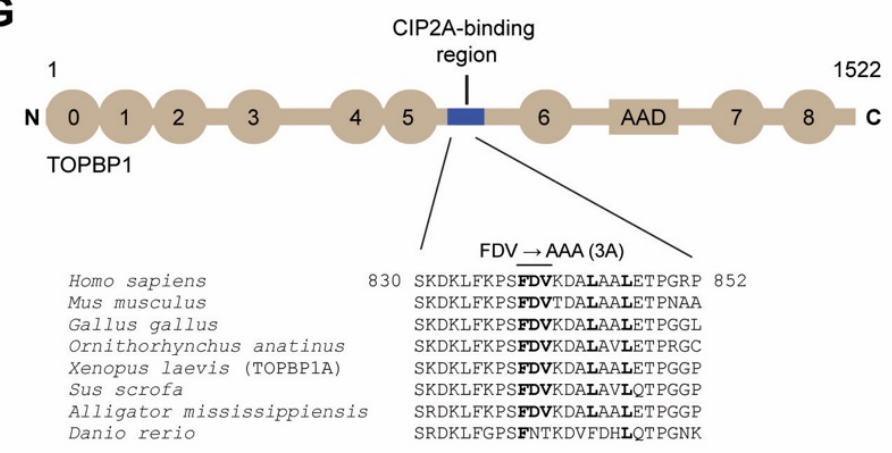

BD-TOPBP $1^{3 A}+$ AD-CIP2A $(1-560) \# 1$
C

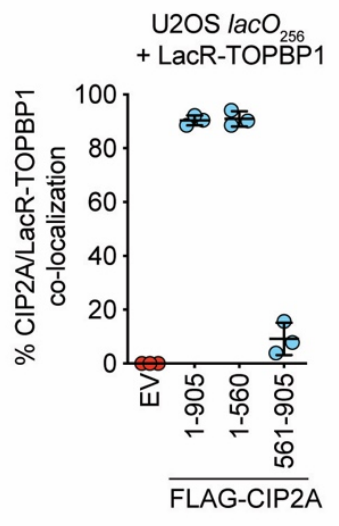

SD - Trp - Leu

(n)

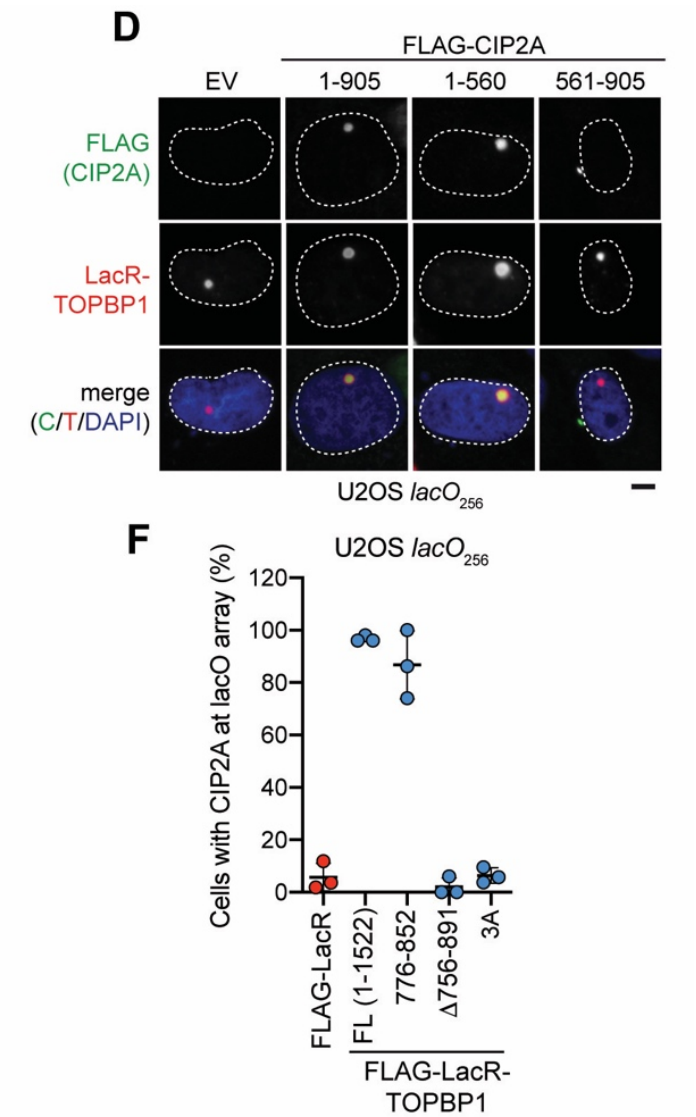

H
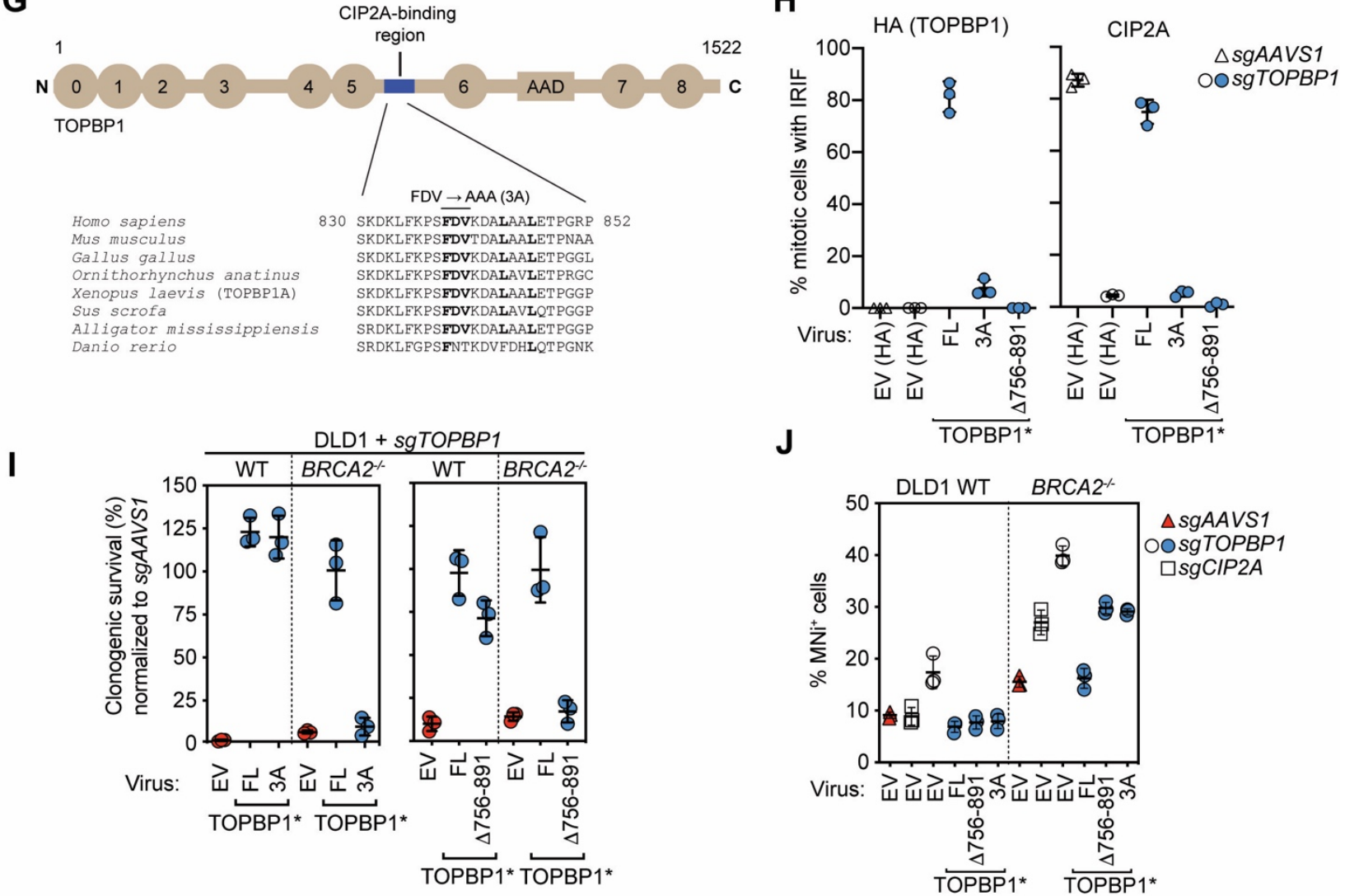

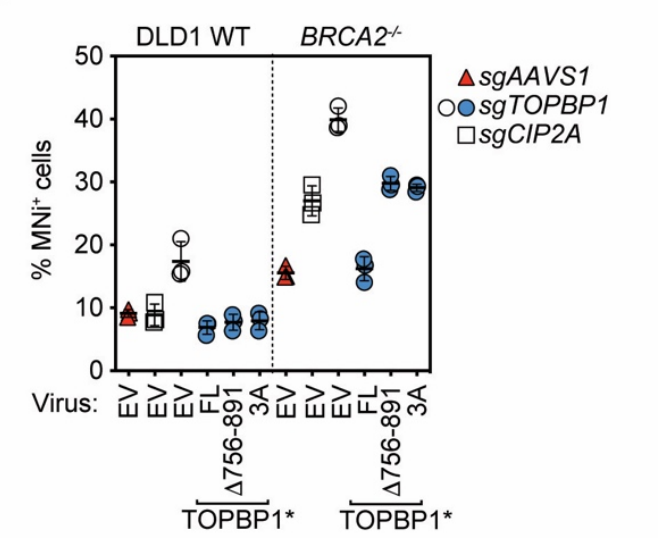


Fig. 4. The CIP2A-TOPBP1 interaction is essential in $B \boldsymbol{R C A} \boldsymbol{2}^{-/-}$cells. (A) Co-immunoprecipitation of CIP2A with TOPBP1. Whole-cell extracts from 293T cells, untreated or treated with nocodazole for $16 \mathrm{~h}$, were subjected to immunoprecipitation with normal mouse IgG or a CIP2A antibody and were then immunoblotted with TOPBP1 (top) or CIP2A (bottom) antibodies. (B) Schematic of the LacR/LacO assay. (C, D) LacR/LacO assay assessing the interaction of Flag-tagged CIP2A and deletion mutants with LacR-TOPBP1 in U2OS $\mathrm{lacO}_{256}$ cells. Quantitation of the assay is in $\mathrm{C}$ where 3 biological replicates are shown and the bars represent the mean \pm S.D. Representative micrographs are shown in D. Scale bar $=10 \mu \mathrm{m}$. (E) Yeast two-hybrid assay for interaction between TOPBP1 variants and CIP2A (1-560). Expression of proteins was verified by immunblotting but not shown. (F) LacR/LacO assay assessing the interaction between endogenous CIP2A and TOPBP1 variants fused to Flag-LacR. Data points represent biological replicates and data is presented as the mean \pm S.D. FL=full-length. (G) Schematic of TOPBP1 and sequence conservation of the minimal CIP2Ainteraction motif. (H) Quantitation of CIP2A and HA-tagged TOPBP1 mitotic foci in DLD1 cells stably expressing full-length (FL) or the indicated mutants of sgRNA-resistant TOPBP1 (TOPBP1*) or empty virus encoding only the HA tag $(\mathrm{EV}(\mathrm{HA}))$ followed by transduction of viruses expressing both Cas9 and sgRNAs targeting TOPBP1 ( $\operatorname{sg} T O P B P 1)$ or AAVS1 ( $s$ AAVS1). Data points represent biological replicates and the bars represent the mean \pm S.D. $(n=3)$. (I) Clonogenic survival of DLD1 wild-type (WT) and $B R C A 2^{-/-}$cells stably expressing sgRNA-resistant TOPBP1 (TOPBP1*, FL), the indicated TOPBP1 mutants, or an empty virus (EV) followed by inactivation of the chromosomal copies of TOPBP1 with an sgRNA and Cas9 ( $\operatorname{sg} T O P B P 1)$. Quantitation of the data is shown in I where representative images of the crystal violet-stained colonies are shown Fig. S4F. Data points represent biological replicates, and the error bars represent the mean \pm S.D. $n=3$. (J) Quantitation of micronuclei $(\mathrm{MNi})$ in DLD1 wild-type (WT) and $B R C A 2^{-/-}$cells stably expressing sgRNA-resistant TOPBP1 (TOPBP1*), the indicated TOPBP1 mutants, or an empty virus (EV) followed by inactivation of TOPBP1,CIP2A or AAVS1 with the indicated sgRNAs and Cas9. Data points represent biological replicates, and the bars represent the mean \pm S.D. $n=3$. 
FIGURE 5

A $_{\text {B6L (DD-TOPBP1 756-1000) }}$

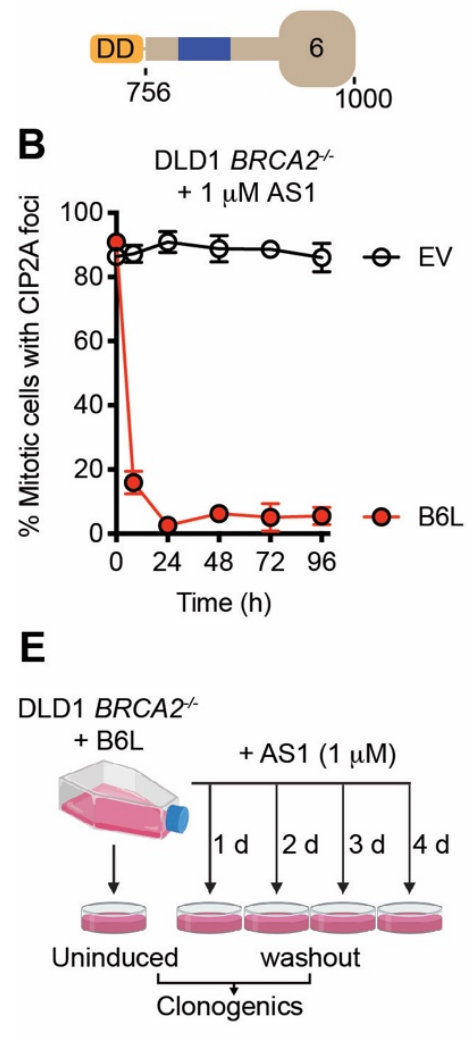

H

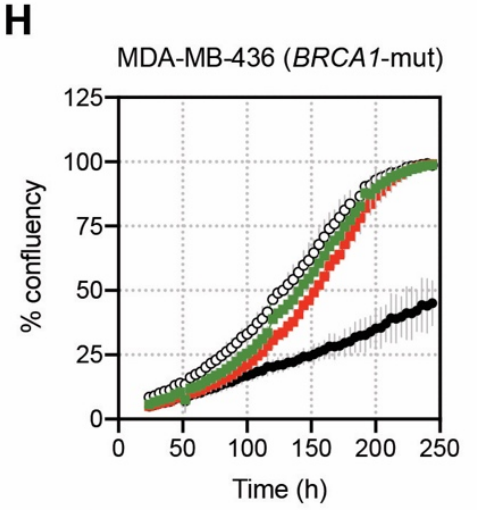

$\rightarrow$ EV uninduced $-0-$ B6L uninduced

$\rightarrow E V+A S 1 \quad \rightarrow B 6 L+A S 1$

C

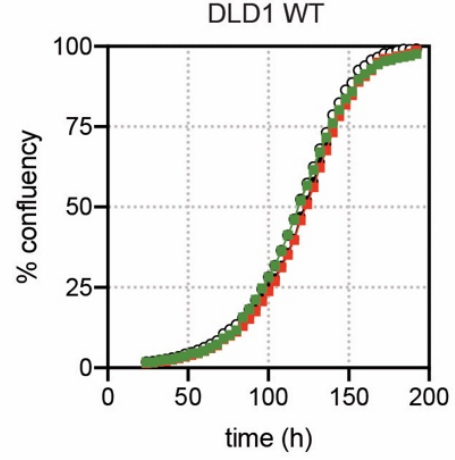

- EV uninduced $-\mathrm{O}-\mathrm{B} 6 \mathrm{~L}$ uninduced

$\rightarrow$ EV + Shield-1 $\rightarrow$ B6L + Shield-1

$\mathbf{F}$

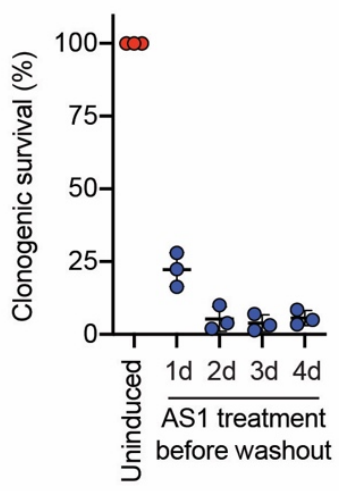

I

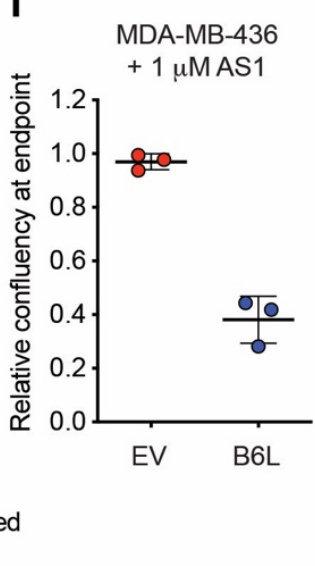

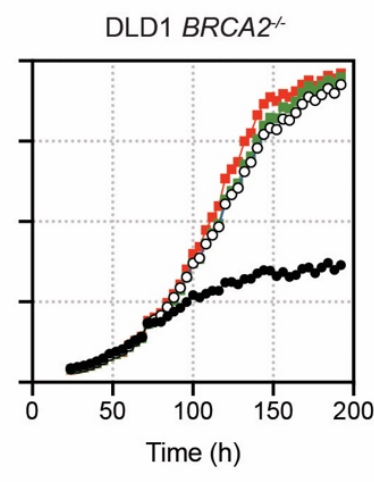

D

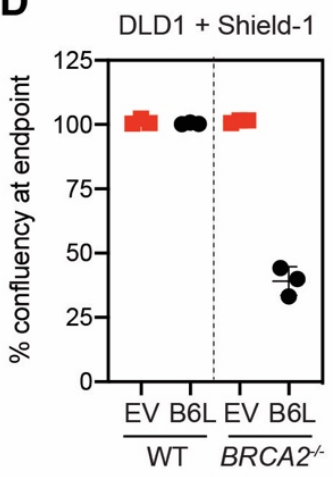

G

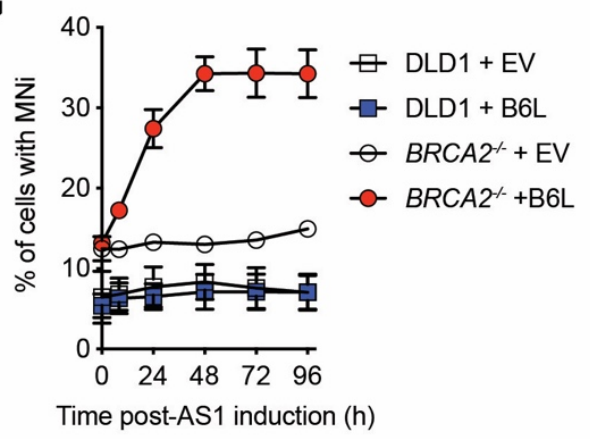

J

DLD1 $B R C A 2^{\prime-~}+\mathrm{B} 6 \mathrm{~L}$

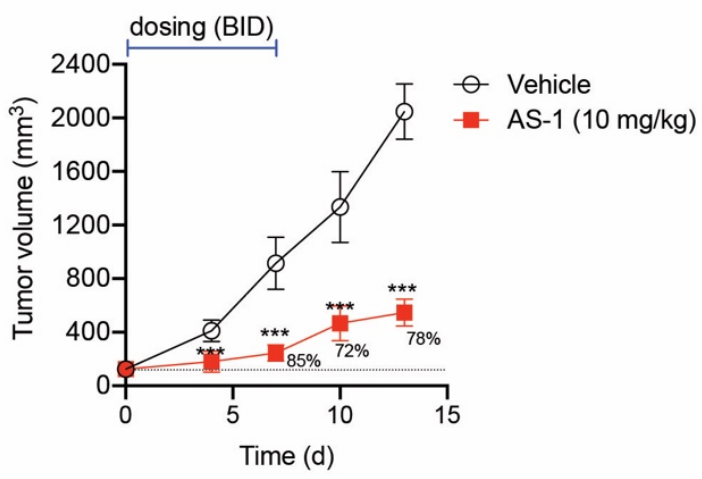

Fig. 5. Therapeutic proof-of-concept. (A) Schematic of B6L, a fragment derived from TOPBP1 residues 756-1000 fused to the destabilization domain (DD). (B) Quantitation of mitotic CIP2A foci in DLD1 BRCA2 $2^{-/}$upon B6L stabilization. Data is shown as mean \pm S.D. $(\mathrm{n}=3)$. (C) Representative proliferation curves for DLD1 parental (left) and $B R C A 2^{-/-}$(right) cells upon B6L stabilization by Shield-1 treatment $(1 \mu \mathrm{M})$. Cells were transduced with an empty virus (EV) that expresses the DD domain as control. (D) Aggregate of 3 biological replicates of the experiment shown in (c). Data is presented as mean \pm S.D. $(n=3)$. (E) Schematic of the experiment shown in F. (F) Clonogenic survival 
of DLD1 $\mathrm{BRCA2}^{-/}$cells following expression of B6L for the indicated periods of time. Data is presented as mean \pm S.D. $(\mathrm{n}=3)$. (G) Quantitation of micronuclei (MNi)-positive cells in DLD1 WT or $B R C A 2^{-/-}$cells following addition of AS1. Data presented as mean \pm S.D. $(\mathrm{n}=3)$. (H) Representative proliferation curves for MDA-MB-436 cells upon B6L stabilization by AS1 treatment ( $1 \mu \mathrm{M})$. Cells were transduced with an empty virus (EV) that expresses the DD domain as control. (I) Aggregate of 3 biological replicates of the experiment shown in H. Data presented as the mean \pm S.D. (J) Growth of tumor xenografts derived from DLD $B R C A 2^{-/-}$cells transduced with a B6L-encoding lentivirus treated intraperitoneally BID for $7 \mathrm{~d}$ either with AS-1 $(10 \mathrm{mg} / \mathrm{kg})$ or with vehicle. After termination of treatment, tumors were grown and monitored without for an additional $8 \mathrm{~d}$. Data is presented at the mean \pm S.D. $(n=8)$. Tumor growth inhibition is indicated, and significance $(* * * p<0.001)$ was determined by a two-tailed unpaired Student's t-test. See Fig. S6 for additional controls and pharmacokinetics of the AS-1 compound. 


\section{Figure S1}

\section{A}

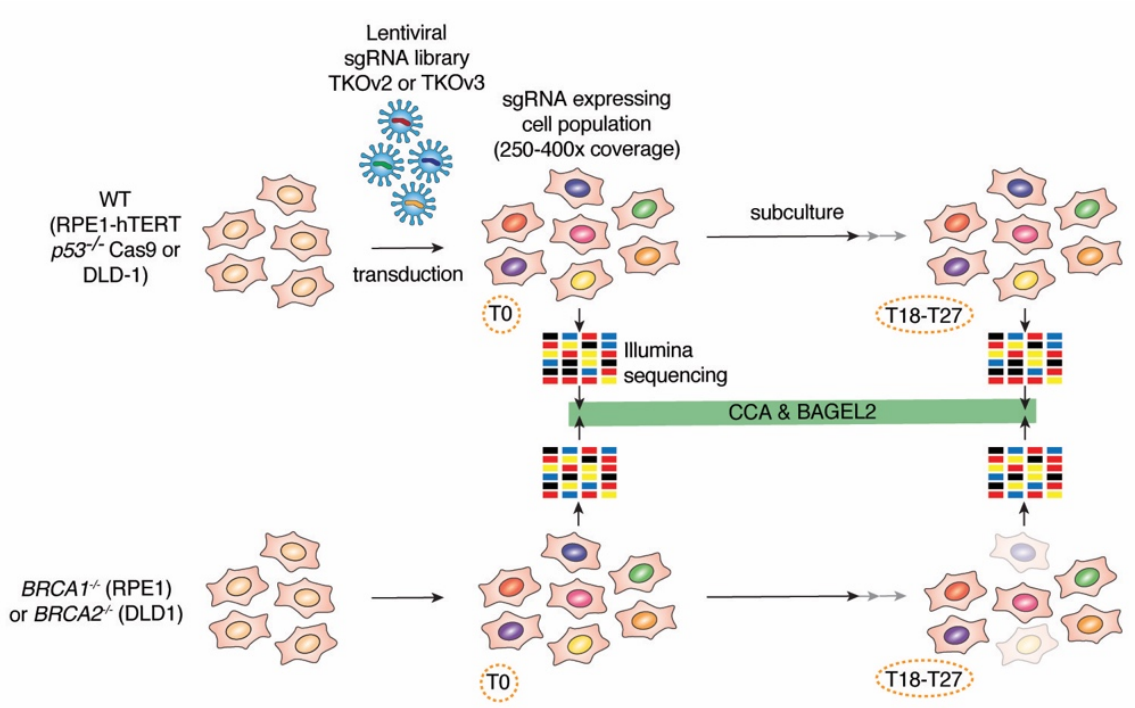

B

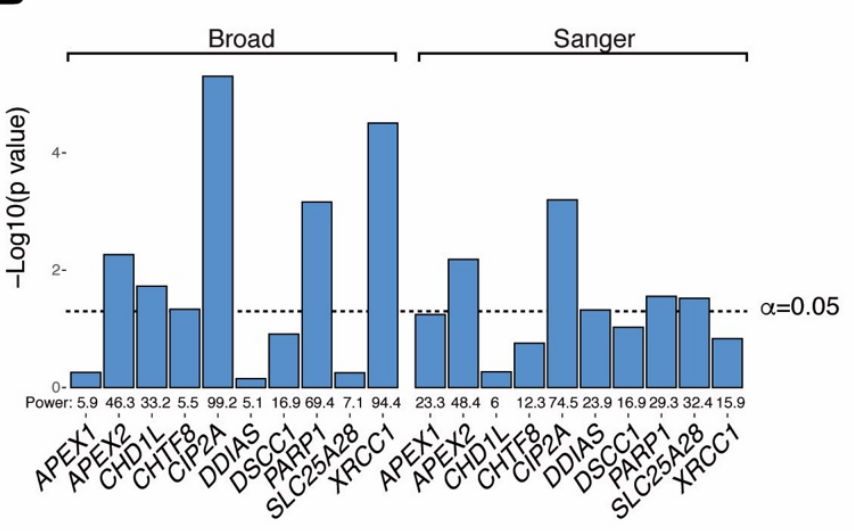

C

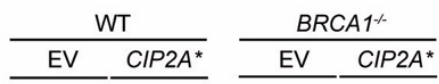

D

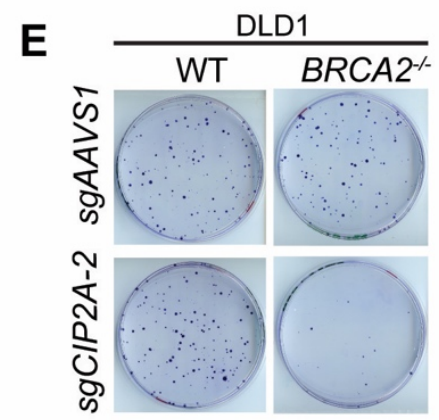

RPE1-hTERT p53- Cas9

Fig. S1. Supporting Data on the identification of $C I P 2 A$ as synthetic lethal with BRCA1- and BRCA2-deficiency. (A) Schematic of the isogenic dropout CRISPR screens to identify syntheticlethal interactions with BRCA1- and BRCA2-deficiency. (B) Statistical analyses for the data shown in Fig. 1BC. Shown are the results of a Mann-Whitney test comparing the values of the BRCAproficient $\left(\mathrm{BRCA}^{+}\right)$and -deficient $\left(\mathrm{BRCA}^{-}\right)$gene depletion scores for the indicated genes. $(\mathbf{C})$ 
Immunoblotting of whole-cell extracts of RPE1-hTERT $p 53^{-/-}$Cas9 cells, parental (WT) or $B R C A 1^{-/-}$ , expressing the indicated sgRNAs and either a virus expressing an sgRNA-resistant $C I P 2 A\left(C I P 2 A^{*}\right)$ fused to a FLAG epitope-coding sequence or an empty virus (EV). Lysates were probed for FLAG (exogenous CIP2A), CIP2A and tubulin (loading control). (D, E) Representative images of the clonogenic survival assays shown in Fig. 1D (D) and the images for the DLD1 WT clonogenics relating to Fig. $1 \mathrm{~F}(\mathrm{E})$. 


\section{Figure S2}

A

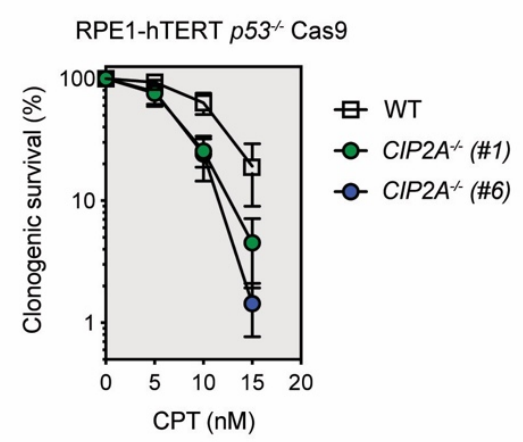

B
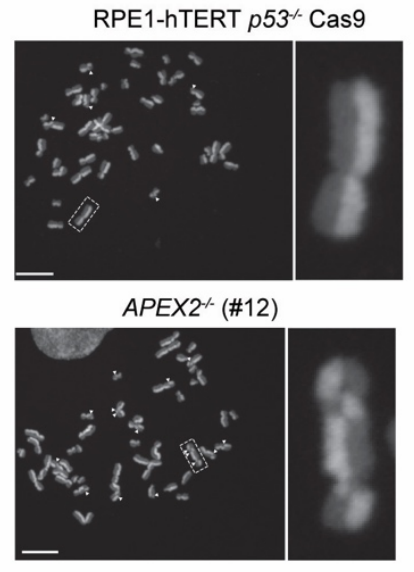

D

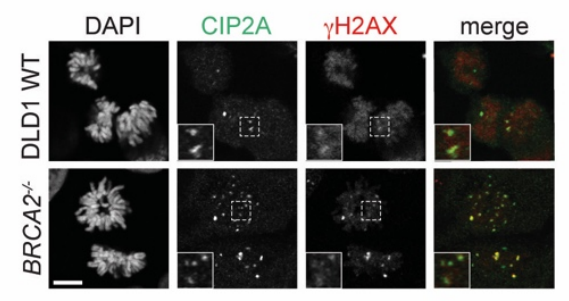

$\mathbf{F}$
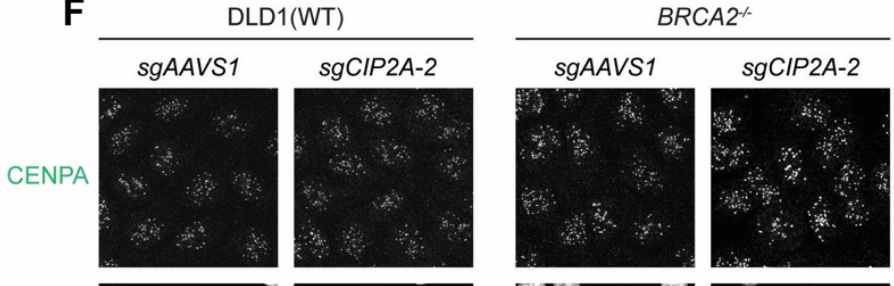

RPE1-hTERT $p 53^{` C}$ Cas9 BRCA1^
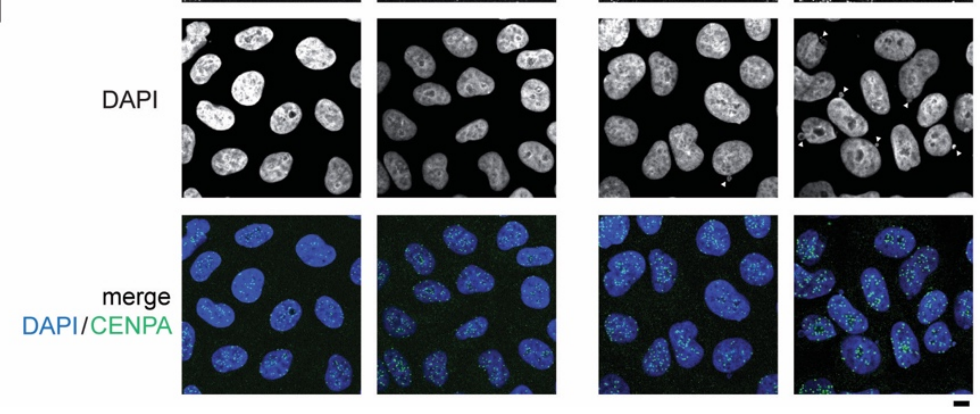

Fig. S2. Loss of CIP2A does not cause DNA lesions requiring HR for their repair. (A) Clonogenic survival assay of RPE1-hTERT $p 53^{-/}$Cas 9 cells of the indicated genotype following treatment with camptothecin (CPT). Data points represent the mean \pm S.D. $(n=3)$. WT=wild type. $($ B $)$ Representative micrographs of metaphase spreads for SCE analysis, relates to Fig. 2A. Arrowheads indicate an SCE event. Scale bar $=10 \mu \mathrm{m}$. (C) Immunofluorescence analysis of isogenic RPE1-hTERT $p 53^{-/}$Cas9derived WT and $C I P 2 A^{-/}$cells with a CIP2A antibody. Scale bar $=10 \mu \mathrm{m}$. (D) Representative micrographs of the experiment shown in Fig. 2G. Analysis of spontaneous CIP2A foci in DLD1 WT 
and $B R C A 2^{-/-}$mitotic cells. Scale bar $=10 \mu \mathrm{m}$. (E) Representative micrographs of the experiment presented in Fig. 2H showing scored radial chromosomes and chromosomes with chromatid breaks. Arrowheads indicate chromosome aberrations. (F) Representative micrographs of the experiment shown in Fig. 2I. White triangles show cells with micronuclei. 
bioRxiv preprint doi: https://doi.org/10.1101/2021.02.08.430060; this version posted February 8, 2021. The copyright holder for this preprint (which was not certified by peer review) is the author/funder, who has granted bioRxiv a license to display the preprint in perpetuity. It is made available under aCC-BY-NC-ND 4.0 International license.

Figure S3

A

Olivieri et al. (2020) correlation network $(>0.68)$

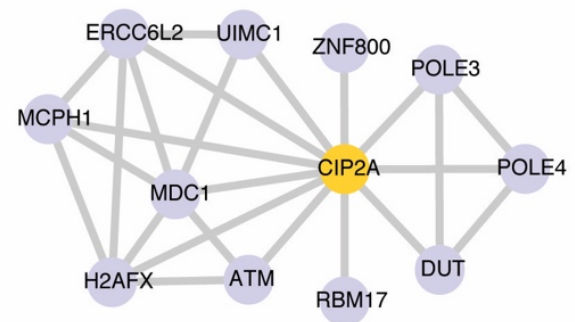

B

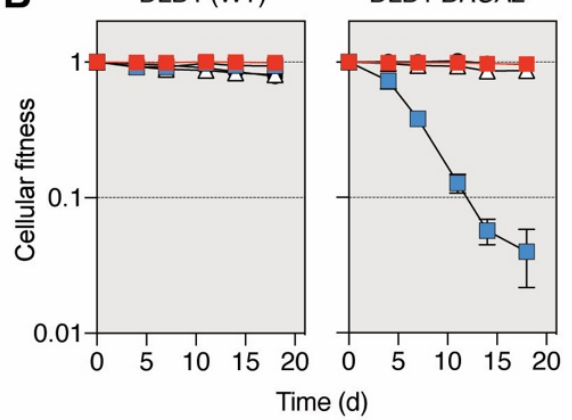

- $\operatorname{sgAAVS1}$

$\triangle \operatorname{sgMDC1-2}$

- $s g M D C 1-3$

$-\operatorname{sgCIP2A-2}$
C

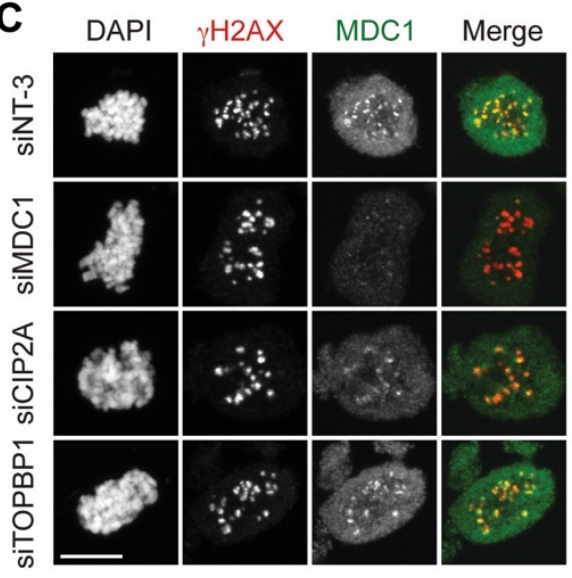

D
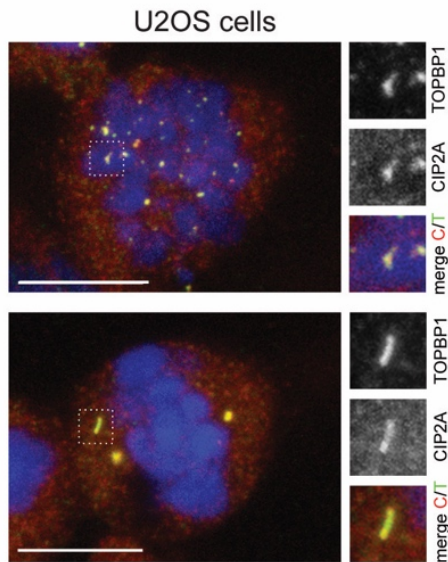

E

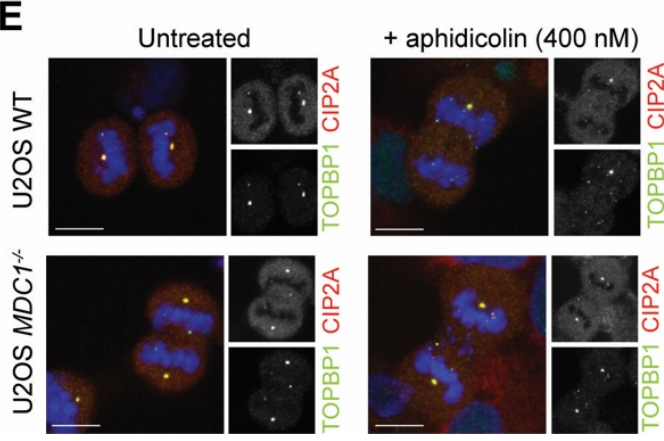

DAPI $\quad \gamma \mathrm{H} 2 \mathrm{AX} \quad \mathrm{CIP} 2 \mathrm{~A} \quad$ Merge

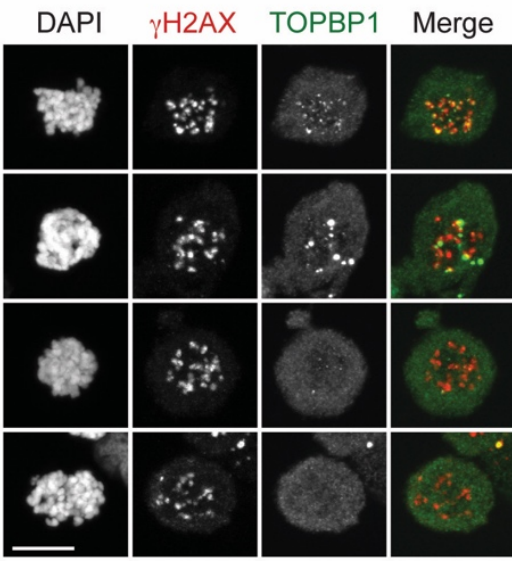

U2OS 1h post-IR (2 Gy)
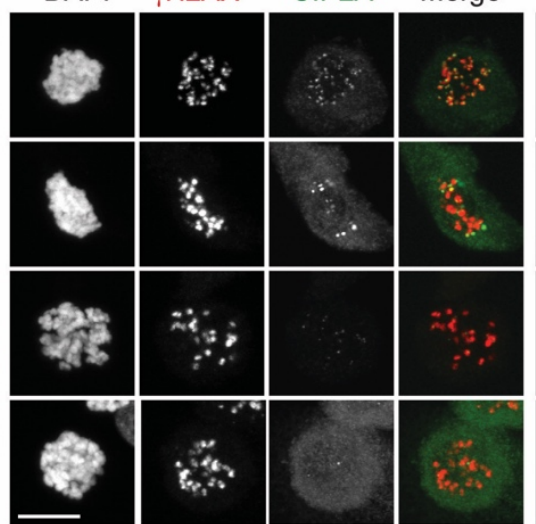

F

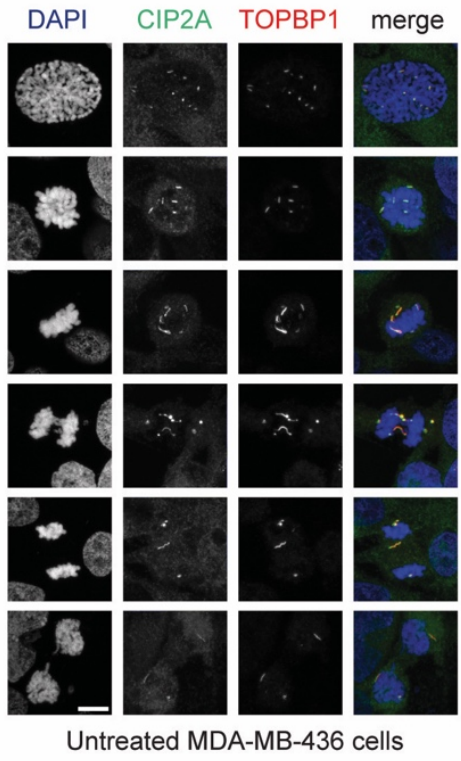


Fig. S3. CIP2A acts in mitosis with TOPBP1. (A) Correlation network based on Pearson correlation of gene-level NormZ derived from the genotoxic dataset shown in ${ }^{21}$. (B) Representative micrographs of the experiment quantitated in Fig. 3c. Nocodazole-treated U2OS cells previously transfected with either a non-targeting siRNA (siNT-3) or the indicated siRNAs were fixed $1 \mathrm{~h}$ post-X-irradiation (2 Gy) and processed for immunofluorescence with the indicated antibodies. Scale bar $=10 \mu \mathrm{m}$. (C) Competitive growth assays in DLD1 Cas9 (WT) or an isogenic $B R C A 2^{-/}$counterpart transduced with virus expressing the indicated sgRNAs. Data are shown as mean \pm S.D. $(n=3$ biologically independent experiments). (D) Additional micrographs of CIP2A/TOPBP1 structures observed in mitotic cells after treatment with low dose aphidicolin. Relates to Fig. 3E. Maximum intensity projections of confocal z-stacks. Scale bar $=10 \mu \mathrm{m}$. Shown here are curved (upper panels) and straight (lower panels) filaments. (E) Maximum intensity projections of confocal z-stacks of U2OS wild type and $M D C 1^{-/}$anaphase cells that were either treated with aphidicolin $(400 \mathrm{nM})$ for $16 \mathrm{~h}$ or left untreated. Scale bars $=10 \mu \mathrm{m}$. Quantitation of this experiment is shown in Fig. 3F. (F) Representative micrographs of the experiment shown in Fig. 3G with additional MDA-MB-436 cells showing elongation of CIP2A-TOPBP1 filaments during mitosis. Maximum intensity projections of confocal Z-stacks are shown. Scale bar $=10 \mu \mathrm{m}$. 
Figure S4

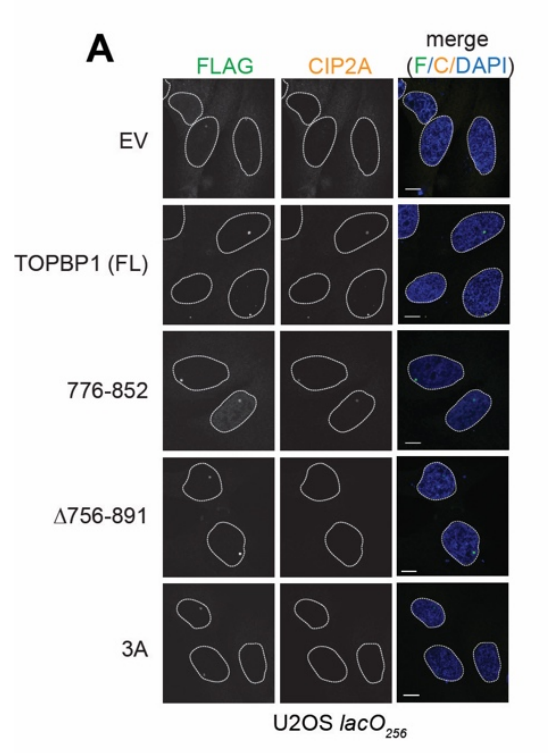

D
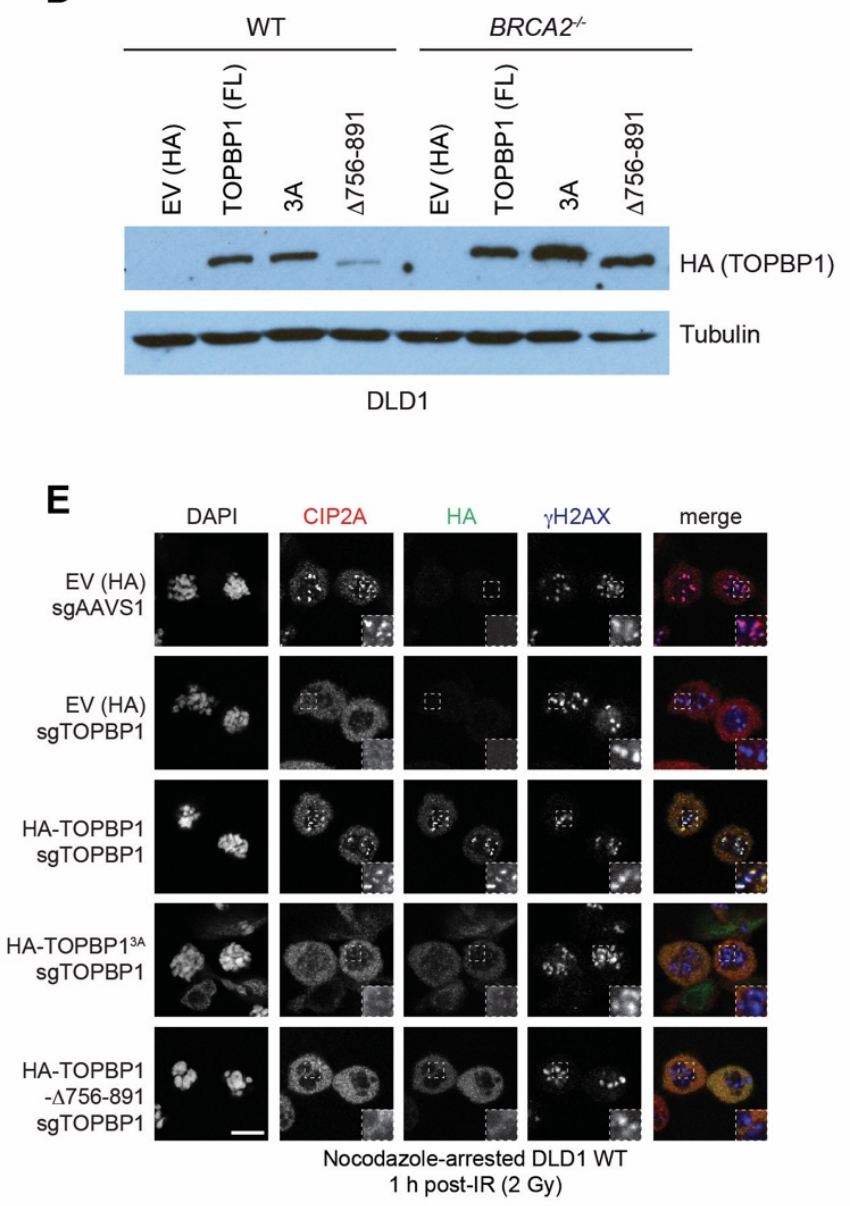

$\mathbf{F}$

C
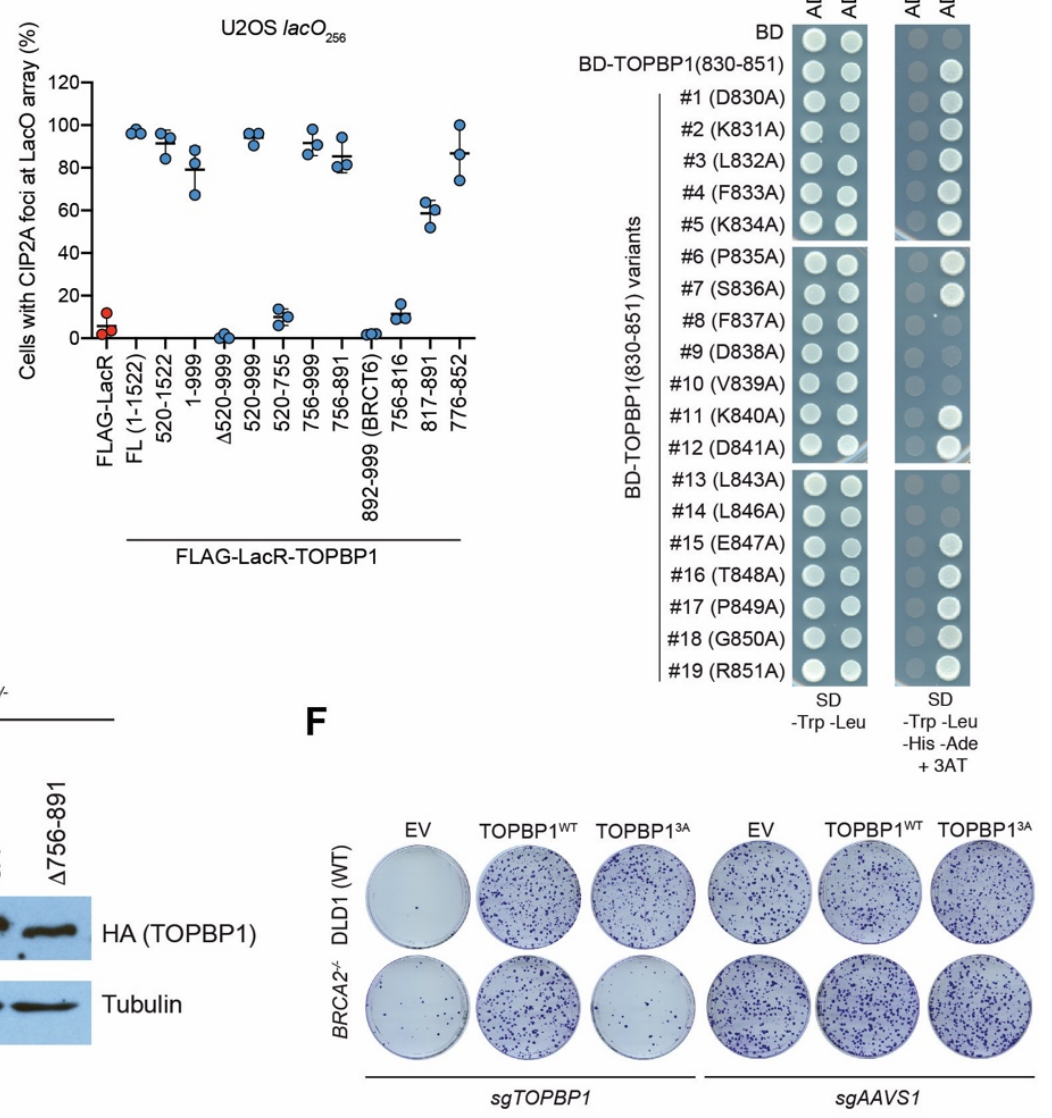

G

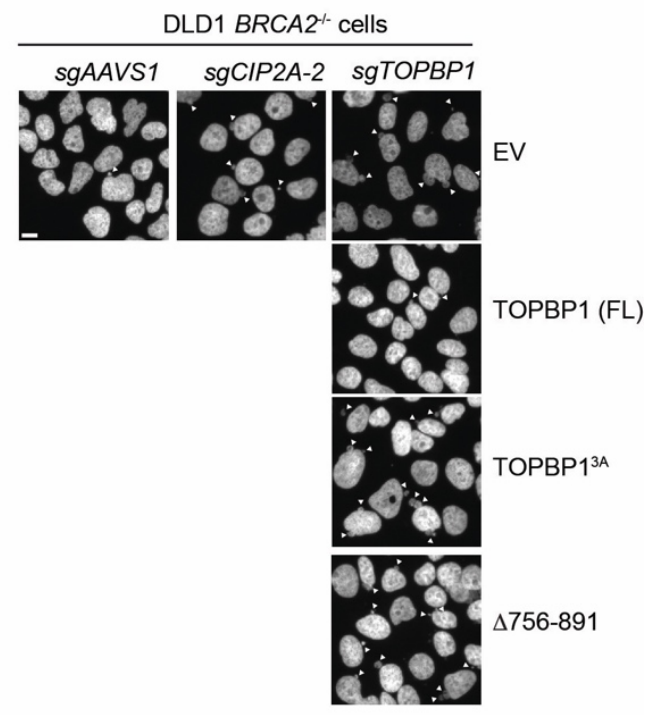

Fig. S4. CIP2A interacts with TOPBP1 to promote BRCA-deficient cell viability. (A) Representative micrographs of the $\mathrm{LacR} / \mathrm{LacO}$ assay assessing the interaction between endogenous CIP2A and TOPBP1 variants fused to Flag-LacR shown in Fig. 4F. Scale bars $=10 \mu \mathrm{m}$. (B) 
LacR/LacO assay assessing the interaction between endogenous CIP2A and TOPBP1 variants fused to Flag-LacR. Data points represent biological replicates, and the bars represent the mean \pm S.D. $(\mathrm{n}=3)$. (C) Alanine scanning of TOPBP1 (830-851) residues by yeast two-hybrid with CIP2A (1-560). These studies identified 5 residues that abolish the TOPBP1-CIP2A interaction when mutated to alanine. $\mathrm{AD}=$ activation domain; $\mathrm{BD}=\mathrm{Ga} 4 \mathrm{DNA}$ binding domain. Expression of proteins was verified by immunblotting but not shown. (D) Immunoblot of whole-cell extracts derived from DLD1 cells transduced with the indicated HA-tagged TOPBP1-expressing lentivirus or an empty virus that expresses an HA epitope (EV(HA)). The lysates were probed with an HA antibody or tubulin (loading control). FL=full-length. (E) Representative micrographs of DLD1 cells transduced with the indicated virus that were arrested in mitosis with a $16 \mathrm{~h}$ treatment with nocodazole, exposed to a 2 Gy IR dose and processed for immunofluorescence with the indicated antibodies $1 \mathrm{~h}$ later. Relates to the experiment quantitated in Fig. 4H. (F) Representative images of the crystal violet stains of the clonogenic survival experiment presented in Fig. 4I. (G) Representative micrographs of the experiment presented in Fig. 4J showing DAPI-stained cells to monitor micronucleation (labeled with arrowheads). Scale bar $=10 \mu \mathrm{m}$. 


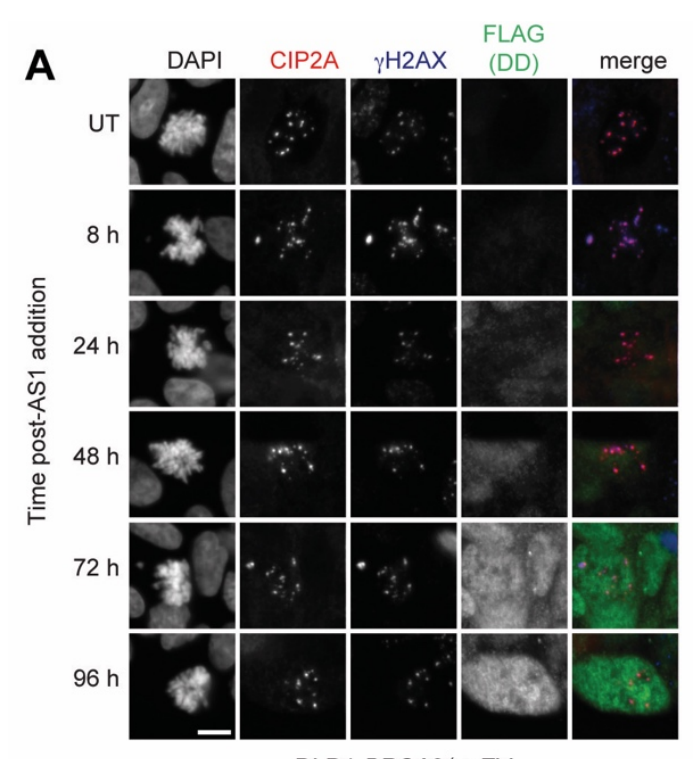

DLD1 BRCA2 - EV

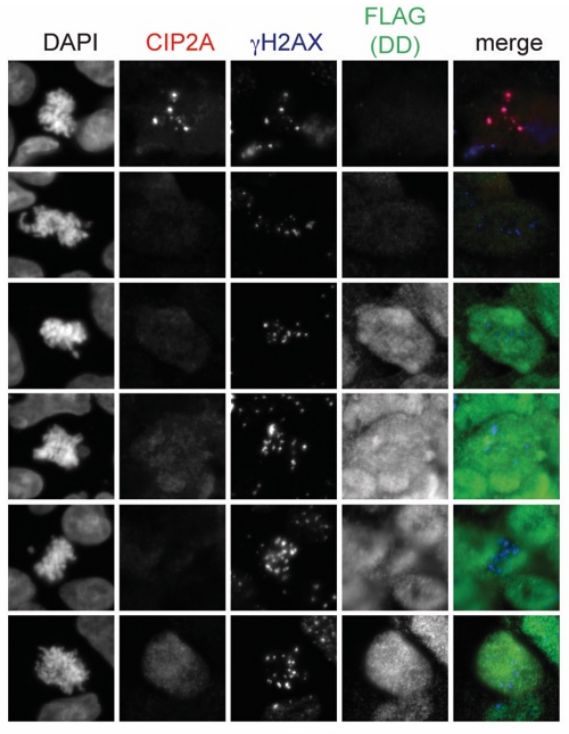

DLD1 BRCA2 $+\mathrm{B} 6 \mathrm{~L}$

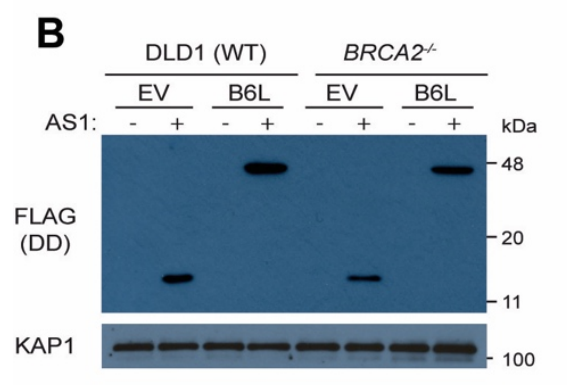

D

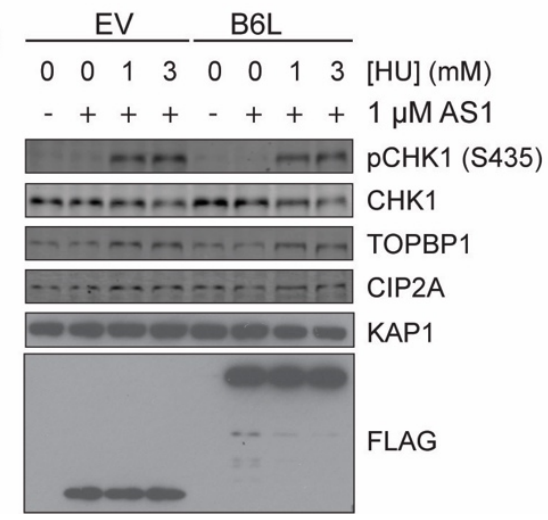

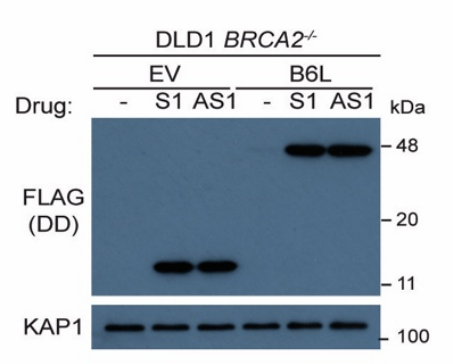

C
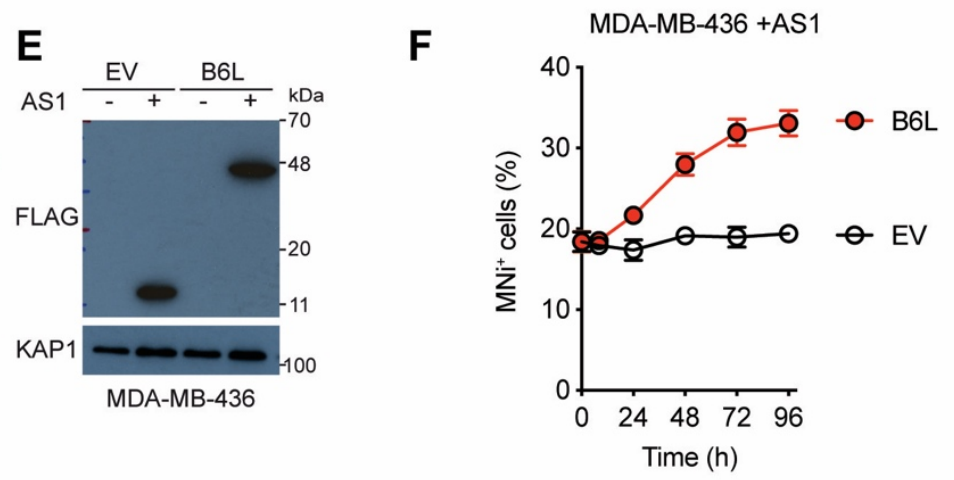

Fig. S5. Disruption of the TOPBP1-CIP2A interaction is lethal in BRCA-deficient cells. (A) DLD1 $B R C A 2^{-/-}$cells transduced with either an empty virus containing only the destabilization domain (DD; EV, left) or a virus encoding B6L were treated with Shield-1 $(1 \mu \mathrm{M})$ for the indicated periods or left untreated (UT). Shown are micrographs of mitotic cells stained for CIP2A, $\gamma \mathrm{H} 2 \mathrm{AX}$ or FLAG (labeling the DD). DNA was stained with DAPI. Scale bar $=10 \mu \mathrm{m}$. Quantitation of the experiment is shown in Fig. 5B. (B) Anti-FLAG immunoblots of whole-cell extracts derived from DLD1 parental (WT) or $B R C A 2^{-/-}$cells treated with either Shield-1 (S1) or Aqua-Shield-1 (AS1) for $72 \mathrm{~h}$. These blots show similar induction of DD (in the empty virus; EV) or B6L upon addition of compound. AntiKAP1 immunoblotting is used as a loading control. (C) Representative images of the clonogenic 
survival experiment presented in Fig. 5F. (D) Immunoblots assessing ATR signaling (CHK1 S345 phosphorylation) in DLD1 cells transduced with either an empty virus (EV) that expresses the unfused DD domain or a virus expressing B6L following induction with AS1. Cells were treated with hydroxyurea (HU) for the indicated times prior to harvesting. e, Anti-FLAG immunoblots of wholecell extracts derived from MDA-MB-436 cells treated with Aqua-Shield-1 (AS1) for 72 h. Anti-KAP1 immunoblotting is used as loading control. (F) Quantitation of the of micronuclei (MNi)-positive cells in MDA-MB-436 transduced with an either empty virus (EV) or B6L-expressing virus following addition of AS1. Data is presented as mean \pm S.D. $(n=3)$. 
Figure S6

A

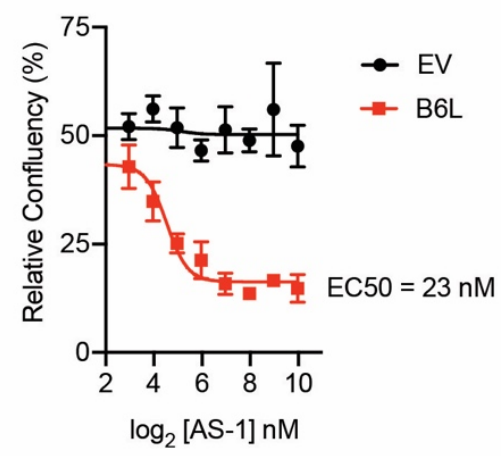

C

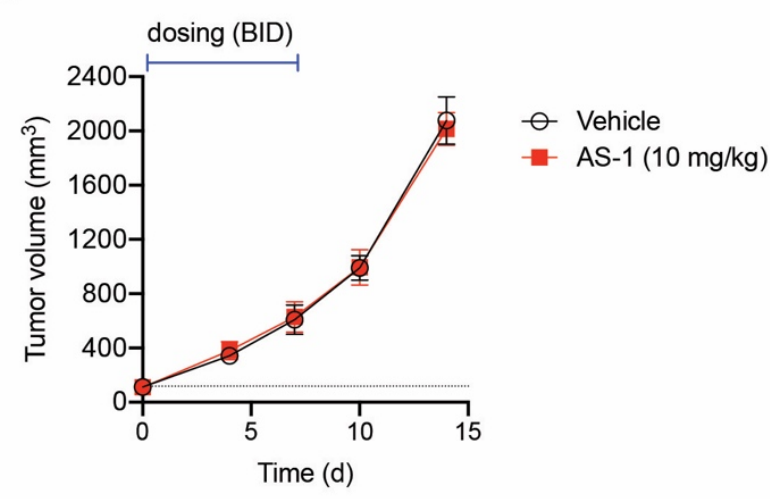

B

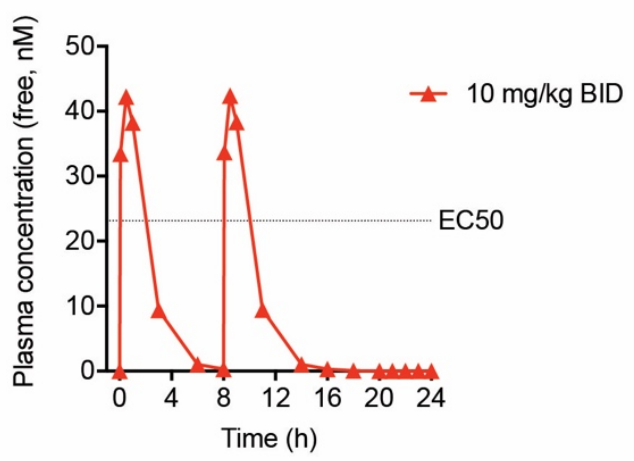

D

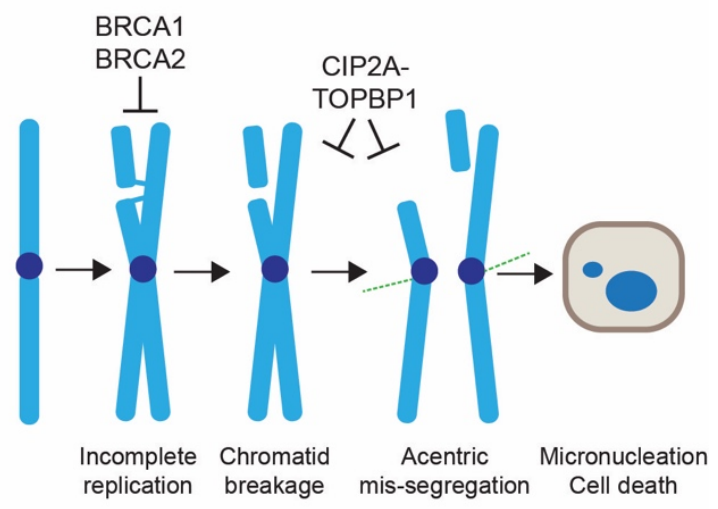

E

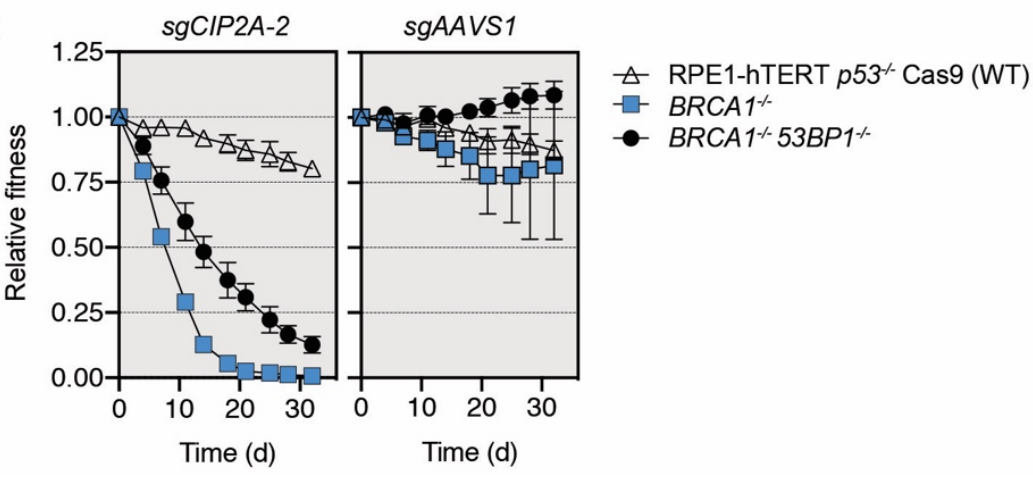

Fig. S6. Control experiments to support the therapeutic hypothesis. (A) Determination of the EC50 growth inhibitory concentration of AS-1 in DLD1 $B R C A 2^{-/}$cells transduced with lentivirus expressing either the DD domain alone (EV) or B6L. Growth was monitored in an Incucyte imager for $7 \mathrm{~d}$. Data is shown as the mean \pm SEM ( $\mathrm{n}=3$ ). (B) Pharmacokinetic analysis of AS-1 free plasma concentration over a $24 \mathrm{~h}$ period in mouse. Data is presented as the mean of values from 3 animals. (C) Growth of tumor xenografts derived from DLD $B R C A 2^{-/-}$cells transduced with a DD-expressing lentivirus (EV) treated with AS-1 $(20 \mathrm{mg} / \mathrm{kg})$ intraperitoneally BID for $7 \mathrm{~d}$ or with vehicle. Data is presented at the mean \pm S.D. $n=8$. This acts as the control for Fig. 5J. (D) Model of the BRCA-CIP2A synthetic lethality. (E) Competitive growth assays in wild-type or RPE1-hTERT $p 53^{-/}$Cas9 (WT) or 
isogenic $B R C A 1^{-/-}$or $B R C A 1^{-/} 53 B P 1^{-/-}$counterparts transduced with virus expressing the indicated sgRNAs. Data are shown as mean \pm S.E.M. ( $\mathrm{n}=3$ biologically independent experiments). Please note that the $53 \mathrm{BPI}^{-/-}$cell line was also subjected to transduction but is not shown for clarity. 


\section{Table S1 (available as separate file)}

Synthetic Lethality screen scores.

\section{Table S2 (available as separate file)}

Raw values used to calculate plots in Fig. 1B,C

\section{Table S3.}

sgRNA used in the study along with primers used for sequencing the edited region in the genome (ICE analysis)

\begin{tabular}{|c|c|c|c|c|}
\hline $\begin{array}{l}\text { targeting } \\
\text { gene }\end{array}$ & $\#$ & $\begin{array}{c}\text { DNA sequence for } \\
\text { sgRNA }\end{array}$ & Forward primer & Reverse primer \\
\hline AAVS1 & & \begin{tabular}{|l} 
5'-GGG GCC ACT \\
AGG GAC AGG AT-3'
\end{tabular} & & \\
\hline APEX2 & 1 & \begin{tabular}{|l} 
5'-AGA TGT TGC \\
GCG TGG TGA GC-3'
\end{tabular} & $\begin{array}{l}\text { 5'-CGG GCC TGG CCA ACT } \\
\text { TCT G-3' }\end{array}$ & $\begin{array}{l}\text { 5'-TAG ATA TGG GGT TTC } \\
\text { GAG AAG GAG-3' }\end{array}$ \\
\hline \multirow{2}{*}{ CIP2A } & 2 & $\begin{array}{l}\text { 5'-CAG TCT ATC AGC } \\
\text { CTG TGC AA-3' }\end{array}$ & $\begin{array}{l}\text { 5'-TGA AAC TAT CCA CAT } \\
\text { TAG CTG TGA G-3' }\end{array}$ & $\begin{array}{l}\text { 5'-GCT GAG TTT GTG GTA } \\
\text { TTT GCT G-3' }\end{array}$ \\
\hline & 3 & $\begin{array}{l}\text { 5'-ATG TTT GAA CAG } \\
\text { TCT CCA CC-3' }\end{array}$ & $\begin{array}{l}\text { 5'-GGA GGA GTT TAA CGT } \\
\text { TGG CTG-3' }\end{array}$ & $\begin{array}{l}\text { 5'-TGG CCT ACT AAA CTT } \\
\text { GGT AGA TGG-3' }\end{array}$ \\
\hline \multirow{2}{*}{ MDC1 } & 2 & $\begin{array}{l}\text { 5'-GCA GAG AGA } \\
\text { CAT CCA GG CGA-3' }\end{array}$ & $\begin{array}{l}\text { 5'-TTT CCC ACT ACA CCT } \\
\text { CGG GA-3' }\end{array}$ & $\begin{array}{l}\text { 5'-GAT TGA TCC TCC AGC } \\
\text { CCC TG-3' }\end{array}$ \\
\hline & 3 & $\begin{array}{l}\text { 5'-CAC CTC GGG } \\
\text { AAG AAT GTG GT-3, }\end{array}$ & $\begin{array}{l}\text { 5-ACC CTG GAC TCA CTG } \\
\text { GAA GT-3' }\end{array}$ & $\begin{array}{l}\text { 5'-GAG AGA CAT CCA GGC } \\
\text { GAT GG-3' }\end{array}$ \\
\hline TOPBP1 & 10 & $\begin{array}{l}\text { 5'-ACT GTC AAA AAA } \\
\text { CCA CTG TG-3' }\end{array}$ & $\begin{array}{l}\text { 5'-TGG AGC CTA TAT GCA } \\
\text { AAC CCA-3' }\end{array}$ & $\begin{array}{l}\text { 5'-TAA GCC CCC AGC TAC } \\
\text { CAT TG-3' }\end{array}$ \\
\hline
\end{tabular}




\section{Table S4.}

Summary of the ICE editing analysis performed in the course of this study.

\begin{tabular}{|c|c|c|c|c|c|c|}
\hline Experiment & Cell line & Genotype & Other vector & $\begin{array}{c}\text { sgRNA } \\
\text { used }\end{array}$ & \begin{tabular}{|c|} 
indel score \\
$(\%)$
\end{tabular} & $\begin{array}{c}\text { KO } \\
\text { score } \\
\text { (\%) }\end{array}$ \\
\hline \multicolumn{7}{|c|}{ Clonogenic survival assays } \\
\hline \multirow{4}{*}{ Related to Fig. 1D } & \multirow{4}{*}{$\begin{array}{l}\text { RPE1 hTERT } \\
\text { Cas9 p53- }\end{array}$} & \multirow{2}{*}{ WT } & \multirow{2}{*}{ NA } & CIP2A-2 & 87 & 52 \\
\hline & & & & CIP2A-3 & 64 & 51 \\
\hline & & \multirow{2}{*}{$B R C A 1^{-/}$} & \multirow{2}{*}{ NA } & CIP2A-2 & 83 & 50 \\
\hline & & & & CIP2A-3 & 69 & 55 \\
\hline \multirow{2}{*}{$\begin{array}{l}\text { Related to Fig. 1F } \\
+21\end{array}$} & \multirow{2}{*}{ DLD-1 } & WT & NA & $C I P 2 A-2$ & 82 & 60 \\
\hline & & $B R C A 2^{-/-}$ & NA & CIP2A-2 & 60 & 46 \\
\hline \multicolumn{7}{|c|}{ Rescue experiments } \\
\hline \multirow{4}{*}{ Related to Fig. 1E } & \multirow{4}{*}{$\begin{array}{l}\text { RPE1 hTERT } \\
\text { Cas9 p53\%- }\end{array}$} & \multirow{2}{*}{ WT } & EV & \multirow{2}{*}{ CIP2A-2 } & 87 & 53 \\
\hline & & & Flag-CIP2A & & 83 & 46 \\
\hline & & \multirow{2}{*}{$B R C A 1^{--}$} & EV & \multirow{2}{*}{ CIP2A-2 } & 87 & 52 \\
\hline & & & Flag-CIP2A & & 80 & 50 \\
\hline \multirow{2}{*}{ Related to Fig. $1 \mathrm{G}$} & \multirow{2}{*}{ DLD-1 } & \multirow{2}{*}{$B R C A 2^{--}$} & EV & \multirow{2}{*}{ CIP2A-2 } & 52 & 42 \\
\hline & & & Flag-CIP2A & & 42 & 34 \\
\hline \multirow{8}{*}{$\begin{array}{c}\text { Related to Fig. } 4 \\
\text { H-J }\end{array}$} & \multirow{8}{*}{ DLD-1 } & \multirow{4}{*}{ WT } & EV & \multirow{8}{*}{$\begin{array}{l}\text { TOPBP1- } \\
10\end{array}$} & 90 & 90 \\
\hline & & & HA-TOPBP1 & & 96 & 96 \\
\hline & & & HA-TOPBP $1^{3 \mathrm{~A}}$ & & 98 & 98 \\
\hline & & & $\begin{array}{c}\text { HA-TOPBP1- } \\
\Delta 756-891 \\
\end{array}$ & & 95 & 95 \\
\hline & & \multirow{4}{*}{$B R C A 2^{-/-}$} & EV & & 81 & 81 \\
\hline & & & HA-TOPBP1 & & 87 & 87 \\
\hline & & & HA-TOPBP $1^{3 A}$ & & 92 & 91 \\
\hline & & & $\begin{array}{c}\text { HA-TOPBP1- } \\
\Delta 756-891\end{array}$ & & 75 & 75 \\
\hline \multicolumn{7}{|c|}{ Two-color growth competition assay } \\
\hline \multirow{3}{*}{$\begin{array}{l}\text { Related to Fig. } \\
\text { S3B }\end{array}$} & \multirow{3}{*}{ DLD-1 Cas9 } & \multirow{3}{*}{ WT } & \multirow{3}{*}{ NA } & $C I P 2 A-2$ & 83 & 68 \\
\hline & & & & $M D C 1-2$ & 92 & 49 \\
\hline & & & & $M D C 1-3$ & 89 & 79 \\
\hline
\end{tabular}


bioRxiv preprint doi: https://doi.org/10.1101/2021.02 08 430060; this version posted February 8,2021 . The copyright holder for this preprint (which was not certified by peer review) is the author/funder, who has granted bioRxiv a license to display the preprint in perpetuity. It is made available under aCC-BY-NC-ND 4.0 International license.

\begin{tabular}{|c|c|c|c|c|c|c|}
\hline & & \multirow{3}{*}{$B R C A 2^{-/-}$} & \multirow{3}{*}{ NA } & CIP2A-2 & 60 & 46 \\
\hline & & & & $M D C 1-2$ & 74 & 53 \\
\hline & & & & $M D C 1-3$ & 63 & 60 \\
\hline \multirow{3}{*}{$\begin{array}{l}\text { Related to Fig. } \\
\text { S6E }\end{array}$} & \multirow{3}{*}{$\begin{array}{l}\text { RPE1 hTERT } \\
\text { Cas9 } p 53^{---}\end{array}$} & WT & \multirow{3}{*}{ NA } & \multirow{3}{*}{ CIP2A-2 } & 82 & 78 \\
\hline & & $B R C A 1^{--}$ & & & 86 & 75 \\
\hline & & $\begin{array}{l}B R C A 1^{-/} \\
53 B P 1^{--}\end{array}$ & & & 91 & 56 \\
\hline
\end{tabular}

\section{Dataset S1. (separate file)}

$B R C A 2^{-/-}$and $C I P 2 A^{-/-}$screen readcounts (TKOv3 library)

\section{Dataset S2. (separate file)}

$B R C A 1^{-/}$screen readcounts (TKOv2 library) 